\title{
Luminescence Spectra of Quantum Dots in Microcavities. II. Fermions.
}

\author{
Elena del Valle \\ Departamento de Física Teórica de la Materia Condensada, \\ Universidad Autónoma de Madrid, Spain, elena.delvalle@uam.es \\ Fabrice P. Laussy \\ School of Physics and Astronomy, University \\ of Southampton, Southampton, United Kingdom \\ Carlos Tejedor \\ Departamento de Física Teórica de la Materia Condensada, \\ Universidad Autónoma de Madrid, Spain
}

(Dated: September 6, 2021) 


\begin{abstract}
We discuss the luminescence spectra of coupled light-matter systems realized with semiconductor heterostructures in microcavities in the presence of a continuous, incoherent pumping, when the matter field is Fermionic. The linear regime - which has been the main topic of investigation both experimentally and theoretically - converges to the case of coupling to a Bosonic material field, and has been amply discussed in the first part of this work. We address here the nonlinear regime, and argue that, counter to intuition, it is better observed at low pumping intensities. We support our discussion with particular cases representative of, and beyond, the experimental state of the art. We explore the transition from the quantum to the classical regime, by decomposing the total spectrum into individual transitions between the dressed states of the light-matter coupling Hamiltonian, reducing the problem to the positions and broadenings of all possible transitions. As the system crosses to the classical limit, rich multiplet structures mapping the quantized energy levels melt and turn to cavity lasing and to an incoherent Mollow triplet in the direct exciton emission for very good structure. Less ideal figures of merit can still betray the quantum regime, with a proper balance of cavity versus electronic pumping.
\end{abstract}

PACS numbers: 42.50.Ct, 78.67.Hc, 42.55.Sa, 32.70.Jz 


\section{INTRODUCTION}

In the first part of this work [1], we have addressed the coupling of light and matter in the particular case where the material excitation follows Bose statistics, solving analytically this so-called linear model of two harmonic oscillators. We emphasized how a proper consideration of the incoherent pumping scheme was needed to describe the effective quantum state realized in the system, and how this bore consequences on the spectral lineshapes, in particular on the ability to resolve a Rabi doublet when the splitting to broadening ratio is small.

In this second part, we turn to the case where the material excitation follows Fermi statistics and explore the Jaynes-Cummings model [2]. These two papers focus particularly on Quantum Dots (QDs) as the matter part of the system, which elementary excitationthe exciton - consists of an electron being promoted from the valence to the conduction band. The coupling of this electron with the vacancy it has created in the valence band (the "hole"), can be either a fermion or a boson, or possibly an interpolating case of the two [3]. Strong Coupling (SC) regime requires efficient coupling of the dot with light, which can be enhanced from the dot point of view by increasing the dipole moment $d$ of the exciton (the coupling strength $g$ is proportional to $|\mathbf{d} \cdot \mathbf{E}|$ where $\mathbf{E}$ is the cavity electric field). Large QDs on the other hand would favor the overlapping of many excitons, whereas small QDs, by confining separately the electron and hole wavefunctions, fully exhibit Pauli blocking [3]. The former case was studied in part I and we now turn to the latter. We use the same formalism and similar techniques, what allows for a comparison and a clear understanding of the specifics of both cases. It is known that in absence of nonlinearity or saturation of some sort, the quantum case is equivalent to the classical one [4]. In particular, the PL spectrum exhibits a Rabi doublet at resonance, which can be equally well accounted for by a purely classical model [5]. There is therefore a strong incentive to evidence nonlinear deviations and attribute them to quantum effects [6, 7, 8, 9, 10]. In studying the coupling of light and matter, be it with atoms or semiconductors, spontaneous emission from a given initial state has been overly privileged as the case of study [11]. Even when the emitter was modelled as a two-level system, this configuration allowed to reduce it to the linear model by considering a single excitation as the initial state [12, 13, 14, 15, 16]. Most of the times that the excitation scheme was considered at the same level as the rest of the 
dynamics, this was for coherent pumping [17, 18, 19, 20, 21, 22, 23]. There has been less considerations for the luminescence spectra under incoherent pumping [24, 25, 26, 27, 28], that is the most adequate to describe semiconductors. In the atomic literature, Löffler et al. [25] have considered spectral shapes for the one-atom laser at resonance by numerical integration of the master equation and in this context have obtained some of the lineshapes of the best system that we study below. Karlovich et al. [28] concentrated on strong coupling at resonance and low pump. In the wake of Part I, we rely on semi-analytical results that put clearly apart the spectral and dynamical aspects of the problem. We shall discuss how our approach allows in general to track the transitions between the different regimes and in particular to identify the quantum to classical one.

One of the most important current task of the SC physics in semiconductors in our view is the quantitative description of the experiment with a theory that can provide statistical estimates to the data, in particular intervals of confidence for the fitting parameters. In this respect, there would be little need for fitting an experiment that would produce a clear observation of the Jaynes-Cummings energy levels, which is a strong qualitative effect. But no such structures have been observed so far and the deviations to Rabi doublet have been understood as non fundamental features of the problem [29]. The most likely reason for this lack of crushing observations of the quantum regime in the PL lineshapes is that the best systems currently available are still beyond the range of parameters that allows the quantum features to neatly dominate. Instead, they are still at a stage where it is easy to overlook more feeble indications, as shall be seen in what follows for less ideal systems that are closer to the experimental situation of today. Another possible reason is that the models are not suitable and a QD cannot be described by a simple two-level system. Then more involved theories should take over, with, e.g., full account for electron and hole band structures and correlations [6, 30, 31]. However, if a simpler theory is successful, notwithstanding the interest of its more elaborate and complete counterpart, it clearly facilitates the understanding and putting the system to useful applications (especially in a quantum information processing context). At present, there is more element of chance left in the research for quantum SC than is actually necessary. If a quantitative description of even a "negative experiment" (not reporting a triplet or quadruplet) could be provided, this would help tracking and probably even direct the progress towards the ultimate goal: a fully understood and controlled SC in the quantum regime. 
The rest of this paper is organized as follow. In Section II, we spell out the model. In Section III, we detail the formalism and provide the expressions for all - and only thosecorrelation functions that enter the problem, making it as computationally efficient as possible for an exact treatment. We provide a decomposition of the final spectra in terms of transitions of the dressed states, which gives a clear physical picture of the problem. In Section IV] we give the analytical expressions for the position and broadening of the resonances of the system at vanishing pumping. Weighting these resonances by the self-consistent dynamics of the system established by finite pumping and decay, gives the final spectral shape. We discuss in particular the notion of SC that varies from manifold to manifold, rather than holding for the entire system as a whole. In Section $\mathrm{V}$, we consider three particular points representative of the experimental situation, plus one point beyond what is currently available. We first discuss their behavior in terms of population and statistical fluctuations as imposed from the pumping conditions. In Section VI, we give the backbone of the final spectra at nonvanishing excitations. This is the numerical counterpart of Section IV] in the presence of arbitrary pumping. In Section VII, we present spectral shapes for the three points in a variety of configuration and compare them to each other. In Section VIII, we investigate the situation at nonzero detuning, which is a case of particular importance in semiconductor physics. Finally, in Section IX, we provide an overview of the results and conclude.

\section{MODEL}

The Hamiltonian that describes a Fermionic QD in strong coupling with a single-mode microcavity is the Jaynes-Cummings Hamiltonian [2] ( $\hbar$ is taken as 1 along the paper):

$$
H=\omega_{a} a^{\dagger} a+\omega_{\sigma} \sigma^{\dagger} \sigma+g\left(a^{\dagger} \sigma+a \sigma^{\dagger}\right)
$$

with $a$ the cavity mode annihilation operator tuned at energy $\omega_{a}$ (obeying Bose statistics) and $\sigma=\left(\sigma_{x}+i \sigma_{y}\right) / 2$ the exciton annihilation operator at energy $\omega_{\sigma}$ (obeying Fermi statistics, $\sigma_{x, y}$ are Pauli matrices). The two modes are coupled with the interaction strength $g$ and close enough to resonance to allow for the rotating wave approximation [32]. The detuning between the modes is defined as $\Delta=\omega_{a}-\omega_{\sigma}$. The Liouvillian to describe the system in the framework of a quantum dissipative master equation, $\partial_{t} \rho=\mathcal{L} \rho$, has the same form as that 
in Part I of this work [1]

$$
\begin{aligned}
\mathcal{L} O=i[O, H] & +\sum_{c=a, \sigma} \frac{\gamma_{c}}{2}\left(2 c O c^{\dagger}-c^{\dagger} c O-O c^{\dagger} c\right) \\
& +\sum_{c=a, \sigma} \frac{P_{c}}{2}\left(2 c^{\dagger} O c-c c^{\dagger} O-O c c^{\dagger}\right),
\end{aligned}
$$

where $\rho$ is the density matrix for the combined Fermi-emitter/cavity system. The only change in both Eqs. (11) and (2) with respect to their counterpart in Ref. [1] is the replacement of the Boson operator $b$ (as it was called in part I) to describe the matter field, by a Fermion operator: $b \leftrightarrow \sigma$. This interchange has far reaching consequences, as will be seen in the following of this text.

We shall not focus on the difference between the spontaneous emission (SE) of an initial state in absence of any pumping, and the steady state (SS) established in presence of this pumping, as we did in part I for the Boson case. SS is the most relevant case for the experimental configuration that we have in mind. Rather than contrasting the SE/SS results, as was done in Part I, we shall therefore contrast the Boson/Fermion cases. For this reason and for concision, we shall not use the "SS" superscript and assume that which of the SE/SS case is assumed is clear from context or from the presence of the time variable $t$.

In the Boson case, the quantum state of the system is not by itself an interesting quantity as most of its features are contained in its reduced density matrices, that are simply and in all cases thermal states with effective temperatures specified by the mean populations of the modes $n_{a}$ and $n_{\sigma}[1]$, defined by:

$$
n_{a}=\left\langle a^{\dagger} a\right\rangle \quad \text { and } \quad n_{\sigma}=\left\langle\sigma^{\dagger} \sigma\right\rangle
$$

For this reason, the higher order correlator

$$
g^{(2)}=\left\langle a^{\dagger} a^{\dagger} a a\right\rangle / n_{a}^{2},
$$

that measures the fluctuations in the photon numbers, does not contain any new information. In the Fermion case however, $g^{(2)}$ becomes nontrivial, because the saturation of the dot provides a nonlinearity in the system that can produce various types of statistics, from the coherent Poisson distributions, encountered in lasers (where the nonlinearity is provided by the feedback and laser gain), to Fock-state statistics, with antibunching, exhibited by systems with a quantum state that has no classical counterpart. The fluctuations in particle 
numbers influence the spectral shape. The full statistics itself is most conveniently obtained from the master equation with elements $\rho_{m, i ; n, j}$ for $m, n$ photons and $i, j$ exciton $(m, n \in \mathbf{N}$, $i, j \in\{0,1\})$. The distribution function of the photon number is simply $\mathrm{p}[n]=\rho_{n, 0 ; n, 0}+$ $\rho_{n, 1 ; n, 1}$.

Rather than to consider the equations of motion for the matrix elements directly, it is clearer and more efficient to consider only elements that are nonzero in the steady state. These are:

$$
\mathrm{p}_{0}[n]=\rho_{n, 0 ; n, 0}, \quad \mathrm{p}_{1}[n]=\rho_{n, 1 ; n, 1} \quad \text { and } \quad \mathrm{q}[n]=\rho_{n, 0 ; n-1,1}
$$

and correspond to, respectively, the probability to have $n$ photons with $\left(\mathrm{p}_{1}\right)$ or without $\left(\mathrm{p}_{0}\right)$ exciton, and the coherence element between the states $|n, 0\rangle$ and $|n-1,1\rangle$, linked by the SC Hamiltonian. Both $p_{0}$ and $p_{1}$ are real. It is convenient to separate $q$ into its real and imaginary parts, $\mathrm{q}[n]=\mathrm{q}_{\mathrm{r}}[n]+i \mathrm{q}_{\mathrm{i}}[n]$ as they play different roles in the dynamics. The equations for these quantities, derived from Schrödinger equation for the Liouvillian Eq. (2), read:

$$
\begin{aligned}
\partial_{t} \mathrm{p}_{0}[n]= & -\left(\left(\gamma_{a}+P_{a}\right) n+P_{a}+P_{\sigma}\right) \mathrm{p}_{0}[n]+\gamma_{a}(n+1) \mathrm{p}_{0}[n+1]+P_{a} n \mathrm{p}_{0}[n-1] \\
& +\gamma_{\sigma} \mathrm{p}_{1}[n]-2 g \sqrt{n} \mathrm{q}_{\mathrm{i}}[n], \\
\partial_{t} \mathrm{p}_{1}[n]= & -\left(\left(\gamma_{a}+P_{a}\right) n+P_{a}+\gamma_{\sigma}\right) \mathrm{p}_{1}[n]+\gamma_{a}(n+1) \mathrm{p}_{1}[n+1]+P_{a} n \mathrm{p}_{1}[n-1] \\
& +P_{\sigma} \mathrm{p}_{0}[n]+2 g \sqrt{n+1} \mathrm{q}_{\mathrm{i}}[n+1], \\
\partial_{t} \mathrm{q}_{\mathrm{i}}[n]= & -\left(\left(\gamma_{a}+P_{a}\right) n-\frac{\gamma_{a}-P_{a}}{2}+\frac{\gamma_{\sigma}+P_{\sigma}}{2}\right) \mathrm{q}_{\mathrm{i}}[n] \\
& +\gamma_{a} \sqrt{(n+1) n} \mathrm{q}_{\mathrm{i}}[n+1]+P_{a} \sqrt{(n-1) n} \mathrm{q}_{\mathrm{i}}[n-1] \\
& +g \sqrt{n}\left(\mathrm{p}_{0}[n]-\mathrm{p}_{1}[n-1]\right)-\Delta \mathrm{q}_{\mathrm{r}}[n], \\
\partial_{t} \mathrm{q}_{\mathrm{r}}[n]= & -\left(\left(\gamma_{a}+P_{a}\right) n-\frac{\gamma_{a}-P_{a}}{2}+\frac{\gamma_{\sigma}+P_{\sigma}}{2}\right) \mathrm{q}_{\mathrm{r}}[n] \\
& +\gamma_{a} \sqrt{(n+1) n} \mathrm{q}_{\mathrm{r}}[n+1]+P_{a} \sqrt{(n-1) n} \mathrm{q}_{\mathrm{r}}[n-1]+\Delta \mathrm{q}_{\mathrm{i}}[n] .
\end{aligned}
$$

Note that in the steady state, Eqs. (6) are detailed-balance type of equations. The conditional photon statistics with and without the exciton are similar, and coupled through the imaginary part of the q distribution (that is not a probability). At resonance, the real part of the coherence distribution, $\mathrm{q}_{\mathrm{r}}$, gets decoupled and vanishes in the steady state. As a result, only Eqs. (6a)-(6c) need to be solved. When $g$ vanishes, $q_{i}$ does not couple the two modes anymore, and their statistics become thermal like in the boson case. Through the 
off-diagonal elements $q_{i}$, the photon density matrix can vary between Poissonian, thermal (superpoissonian) and subpoissonian distributions [33].

\section{CORRELATION FUNCTIONS AND SPECTRA}

The main quantity of interest of this paper is the luminescence spectrum of the system. In the Boson case, the symmetry $a \leftrightarrow b$ allowed to focus exclusively on the cavity-emission without loss of generality, as the direct exciton emission could be obtained from the cavity emission by interchanging parameters. Here, the exciton (Fermion) and photon (Boson) are intrinsically different, and no simple relationship links them. They must therefore be computed independently:

$$
S_{c}(\omega)=\frac{1}{\pi n_{c}} \lim _{t \rightarrow \infty} \Re \int_{0}^{\infty}\left\langle c^{\dagger}(t) c(t+\tau)\right\rangle e^{i \omega \tau} d \tau, \quad \text { with } c=a, \sigma .
$$

We consider normalized spectra for convenient comparisons of the lineshapes. The normalizing factor is the population $n_{c}$, as seen straightforwardly from $\int S_{c}(\omega) d \omega=1$.

As in Part I, we recourse to the quantum regression theorem to compute the two times average $\left\langle c^{\dagger}(t) c(t+\tau)\right\rangle$. We first identify the set of closing operators $C_{\{\eta\}}$ in the sense that, for any operator $\Omega$, the relation $\operatorname{Tr}\left(C_{\{\eta\}} \mathcal{L} \Omega\right)=\sum_{\{\lambda\}} M_{\{\eta \lambda\}} \operatorname{Tr}\left(C_{\{\lambda\}} \Omega\right)$ is satisfied for some $M_{\{\eta \lambda\}}$ to be specified. In the linear case, the set of $a^{m} b^{n}$ with $m, n \in \mathbf{N}$ is closed, what allows for an analytical solution. In the nonlinear case, four indices are required to label the closing operators, namely $\{\eta\}=(m, n, \mu, \nu)$ in $C_{\{\eta\}}=a^{\dagger^{m}} a^{n} \sigma^{\dagger^{\mu}} \sigma^{\nu}$ with $m, n \in \mathbf{N}$ and $\mu$, $\nu \in\{0,1\}$. The links established between them by the Liouvillian dynamics are given by $\operatorname{Tr}\left(a^{\dagger^{m}} a^{n} \sigma^{\dagger^{\mu}} \sigma^{\nu} \mathcal{L} \Omega\right)=\sum_{p q \pi \theta} M_{p q \pi \theta} \operatorname{Tr}\left(a^{\dagger^{p}} a^{q} \sigma^{\dagger^{\pi}} \sigma^{\theta} \Omega\right)$, with $M$ defined as:

$$
\begin{aligned}
& \underset{m n \mu \nu}{M m n \mu \nu}=i \omega_{a}(m-n)+i \omega_{\sigma}(\mu-\nu)-\frac{\gamma_{a}-P_{a}}{2}(m+n)-\frac{\gamma_{\sigma}+P_{\sigma}}{2}(\mu+\nu), \\
& M_{m-1, n-1, \mu \nu}^{m n \mu \nu}=P_{a} m n, \quad M_{m n, 1-\mu, 1-\nu}=P_{\sigma} \mu \nu, \\
& M_{m-1, n, 1-\mu, \nu}^{m n \mu \nu}=M_{n, m-1, \nu, 1-\mu}^{*}=i g m(1-\mu), \\
& M_{m, n+1, \mu, 1-\nu}^{m n \mu \nu}=M_{n+1, m, 1-\nu, \mu}^{*}=-i g \nu, \\
& M_{\substack{m m n \mu \mu \\
m, n+1,1-\mu, \nu}}=M_{n+1, m, \nu, 1-\mu}^{*}=2 i g \nu(1-\mu),
\end{aligned}
$$

and zero everywhere else. 
We are interested in this text in $\Omega=c^{\dagger}$ with $c=a$ and $\left\{\eta_{a}\right\}=(0,1,0,0)$ on the one hand, to get the equation for $\left\langle a^{\dagger}(t) a(t+\tau)\right\rangle$ that will provide the cavity emission spectrum, and $c=\sigma$ with $\left\{\eta_{\sigma}\right\}=(0,0,0,1)$ on the other hand, to get the equation for $\left\langle\sigma^{\dagger}(t) \sigma(t+\tau)\right\rangle$ for the QD direct emission spectrum. Contrary to the Boson case, this procedure leads to an infinite set of coupled equations. The equations of motion for both $\left\langle a^{\dagger}(t) C_{(0,1,0,0)}(t+\tau)\right\rangle$ and $\left\langle\sigma^{\dagger}(t) C_{(0,0,0,1)}(t+\tau)\right\rangle$ involve the same family of closing operators $C_{\{\eta\}}$, namely with $\eta \in$ $\bigcup_{k \geq 1} \mathcal{N}_{k}$ where $\mathcal{N}_{1}=\{(0,1,0,0),(0,0,0,1)\}$ the manifold of the boson case, and for $k>1$ :

$$
\mathcal{N}_{k}=\{(k-1, k, 0,0),(k-1, k-1,0,1),(k-2, k, 1,0),(k-2, k-1,1,1)\} .
$$

The links between the various correlators tracked through the indices $\{\eta\}$, are shown in Fig. 1. To solve the differential equations of motion, the initial value of each correlator is also required, e.g., $\left\langle a^{\dagger}(t) a(t+\tau)\right\rangle$ demands $\left\langle\left(a^{\dagger} a\right)(t)\right\rangle$, etc. The initial values of $\left\langle a(t) C_{\{\eta\}}(t+\tau)\right\rangle$ (resp., $\left.\left\langle\sigma(t) C_{\{\eta\}}(t+\tau)\right\rangle\right)$ can be conveniently computed within the same formalism, recurring to $\Omega=1$ and $C_{\{\tilde{\eta}\}}$ with $\{\tilde{\eta}\}=\{m+1, n, \mu, \nu\}$ (resp., $\{m, n, \mu+1, \nu\}$ ). This allows to compute also the single-time dynamics $\left\langle C_{\{\tilde{\eta}\}}(t)\right\rangle$, and their steady state, from the same tools used as for the two-time dynamics through the quantum regression theorem. The indices $\{\tilde{\eta}\}$ required for the single-time correlators form a set- that we call $\tilde{\mathcal{N}}=\bigcup_{k \geq 1} \tilde{\mathcal{N}}_{k}$ that is disjoint from $\bigcup_{k \geq 1} \mathcal{N}_{k}$, required for the two-times dynamics. The set $\tilde{\mathcal{N}}$ has-beside the constant term $\left\{\eta_{0}\right\}=(0,0,0,0)$ - two more elements for the lower manifold (of the Boson case). This is because $\left\{\eta_{a}\right\}=(0,1,0,0)$ and $\left\{\eta_{\sigma}\right\}=(0,0,0,1)$ invoke $(1,1,0,0)$ and $(1,0,0,1)$ for the cavity spectrum on the one hand, and $(0,1,1,0)$ and $(0,0,1,1)$ for the exciton emission on the other. At higher orders $k>1$, all two-times correlators $\mathcal{N}_{k}$ otherwise depend on the same four single-time correlators $\tilde{\mathcal{N}}_{k}$. Independently of which spectrum one wishes to compute, these four elements $(1,1,0,0),(1,0,0,1),(0,1,1,0)$ and $(0,0,1,1)$ of $\tilde{\mathcal{N}}_{1}$ are needed in all cases as they are linked to each other, as shown on Fig. 1.

On the figure, only the type of coupling - coherent, through $g$, or incoherent, through the pumpings $P_{a, \sigma}$-has been represented. Weighting coefficients are given by Eqs. (8). Of particular relevance is the self-coupling of each correlator to itself, not shown on the figure for clarity. Its coefficient, Eq. (8ad), lets enter $\gamma_{a, \sigma}$ that do not otherwise couple any one correlator to any of the others. This makes it possible to describe decay by simply providing an imaginary part to the Energy in Eq. (1). The incoherent pumping, on the other hand, establishes a new set of connections between correlators. Note, however, that 
at the exception of $\left\{\eta_{0}\right\}$, the pumping does not enlarge the sets $\cup \mathcal{N}_{k}, \cup \tilde{\mathcal{N}}_{k}$ : the structure remains the same (also, technically, the computational complexity is identical), only with the correlators affecting each other differently. The addition of $\left\{\eta_{0}\right\}$ by the pumping terms bring the same additional physics in the Boson and Fermion cases: it imposes a self-consistent steady state over a freely chosen initial condition. In the Boson case, the pumping had otherwise only a direct influence in renormalizing the self-coupling of each correlator. In the Fermion case, it brings direct modifications to the Jaynes-Cummings coherent dynamics. But its contribution to the self-coupling is also important, and gives rise to an interesting fermionic opposition to the bosonic effects as seen in Eq. (8a) in the effective linewidth:

$$
\Gamma_{a}=\gamma_{a}-P_{a}, \quad \Gamma_{\sigma}=\gamma_{\sigma}+P_{\sigma}
$$

For later convenience, we also define:

$$
\Gamma_{ \pm}=\frac{\Gamma_{a} \pm \Gamma_{b}}{4}
$$

In Eq. (10), it is seen that, whereas the incoherent cavity pumping narrows the linewidth, as a manifestation of its boson character, the incoherent exciton pumping broadens it. This opposite tendencies bear a capital importance for the lineshapes, as narrow lines favor the observation of a structure, whereas broadening hinders it. On the other hand, the cavity incoherent pumping always results in a thermal distribution of photons with large fluctuations of the particle numbers, that result in an inhomogeneous broadening, whereas the exciton pumping can grow a Poisson-like distribution with little fluctuations. Both types of pumping, however, ultimately bring decoherence to the dynamics and induce the transition into weak coupling (WC), with the lines composing the spectrum collapsing into one. Putting all these effects together, there is an optimum configuration of pumpings where particle fluctuations compensate for the broadening of the interesting lines, enhancing their resolution in the spectrum, as we shall see when we discuss the results below.

As there is no finite closure relation, some truncation is in order. We will adopt the scheme where a maximum of $n_{\mathrm{t}}$ excitation(s) (photon plus excitons) is allowed at the $n_{\mathrm{t}}$ th order, thereby truncating into manifolds of excitations, which is the most relevant picture. The exact result is recovered in the limit $n_{t} \rightarrow \infty$. As seen in Fig. 1, the number $s_{\mathrm{t}}$ of two-time correlators from $\mathcal{N}$ up to order $n_{\mathrm{t}}$ is $s_{\mathrm{t}}=4 n_{\mathrm{t}}-2$ and the number of mean values from $\tilde{\mathcal{N}}$ is $4 n_{\mathrm{t}}$. The problem is therefore computationally linear in the number of excitations, 
and as such is as simple as it could be for a quantum system. The general case consists in a linear system of $s_{\mathrm{t}}$ coupled differential equations, whose matrix of coefficients [specified by Eqs. (8)] is, in the basis of $C_{\{\eta\}}$, a $s_{\mathrm{t}} \times s_{\mathrm{t}}$ square matrix that we denote $\mathbf{M}$. With these definitions, the quantum regression theorem becomes:

$$
\partial_{\tau} \mathbf{v}_{c}(t, t+\tau)=\mathbf{M} \mathbf{v}_{c}(t, t+\tau)
$$

where $\mathbf{v}_{c}(t, t+\tau)=\left\langle c^{\dagger}(t) \mathbf{C}_{\{\eta\}}(t+\tau)\right\rangle$. Explicitly, for the lower manifolds, e.g., for $c=a$ :

$$
\mathbf{C}_{\{\eta\}}=\left(\begin{array}{c}
C_{(0,1,0,0)} \\
C_{(0,0,0,1)} \\
C_{(1,2,0,0)} \\
C_{(1,1,0,1)} \\
C_{(0,2,1,0)} \\
\vdots
\end{array}\right) \text { and } \mathbf{v}_{a}(t, t+\tau)=\left(\begin{array}{c}
\left\langle a^{\dagger}(t) a(t+\tau)\right\rangle \\
\left\langle a^{\dagger}(t) \sigma(t+\tau)\right\rangle \\
\left\langle a^{\dagger}(t)\left(a^{\dagger} a^{2}\right)(t+\tau)\right\rangle \\
\left\langle a^{\dagger}(t)\left(a^{\dagger} a \sigma\right)(t+\tau)\right\rangle \\
\left\langle a^{\dagger}(t)\left(a^{2} \sigma^{\dagger}\right)(t+\tau)\right\rangle \\
\vdots
\end{array}\right) .
$$

The ordering of the correlators is arbitrary. We fix it to that of Fig. 1, as seen in Eq. (13). With this convention, the indices of the two correlators of interests are:

$$
i_{a}=1, \quad i_{\sigma}=2
$$

To solve Eq. (12), we introduce the matrix $\mathbf{E}$ of normalized eigenvectors of $\mathbf{M}$, and $-\mathbf{D}$ the diagonal matrix of eigenvalues:

$$
-\mathrm{D}=\mathrm{E}^{-1} \mathrm{ME} .
$$

The formal solution is then $\mathbf{v}_{c}(t, t+\tau)=\mathbf{E} e^{-\mathbf{D} \tau} \mathbf{E}^{-1} \mathbf{v}_{c}(t, t)$. Integration of $\int e^{(-\mathbf{D}+i \omega) \tau} d \tau$ and application of the Wiener-Khintchine formula yield for the $i_{a}$ th and $i_{\sigma}$ th rows of $\mathbf{v}_{c}$ the emission spectra of the cavity, $S_{a}=\frac{1}{\pi n_{a}} \Re \int\left\langle a^{\dagger}(t) a(t+\tau)\right\rangle e^{i \omega \tau} d \tau$, and of the direct exciton emission, $S_{\sigma}=\frac{1}{\pi n_{\sigma}} \Re \int\left\langle\sigma^{\dagger}(t) \sigma(t+\tau)\right\rangle e^{i \omega \tau} d \tau$, respectively. We find, to order $n_{\mathrm{t}}$ :

$$
S_{c}(\omega)=\frac{1}{\pi} \Re \sum_{p=1}^{s_{\mathrm{t}}} \frac{L_{i_{c} p}^{c}+i K_{i_{c} p}^{c}}{D_{p}-i \omega}, \quad c=a, \sigma
$$

where $L_{i_{c} p}^{c}$ and $K_{i_{c} p}^{c}$ are the real and the imaginary part, respectively, of $[\mathbf{E}]_{i_{c} p}\left[\mathbf{E}^{-1} \mathbf{v}_{c}(t, t)\right]_{p} / n_{c}:$

$$
L_{i_{c} p}^{c}+i K_{i_{c} p}^{c}=\frac{1}{n_{c}}[\mathbf{E}]_{i_{c} p} \sum_{q=1}^{s_{\mathrm{t}}}\left[\mathbf{E}^{-1}\right]_{p q}\left[\mathbf{v}_{c}(t, t)\right]_{q}, \quad 1 \leq p \leq s_{\mathrm{t}},
$$




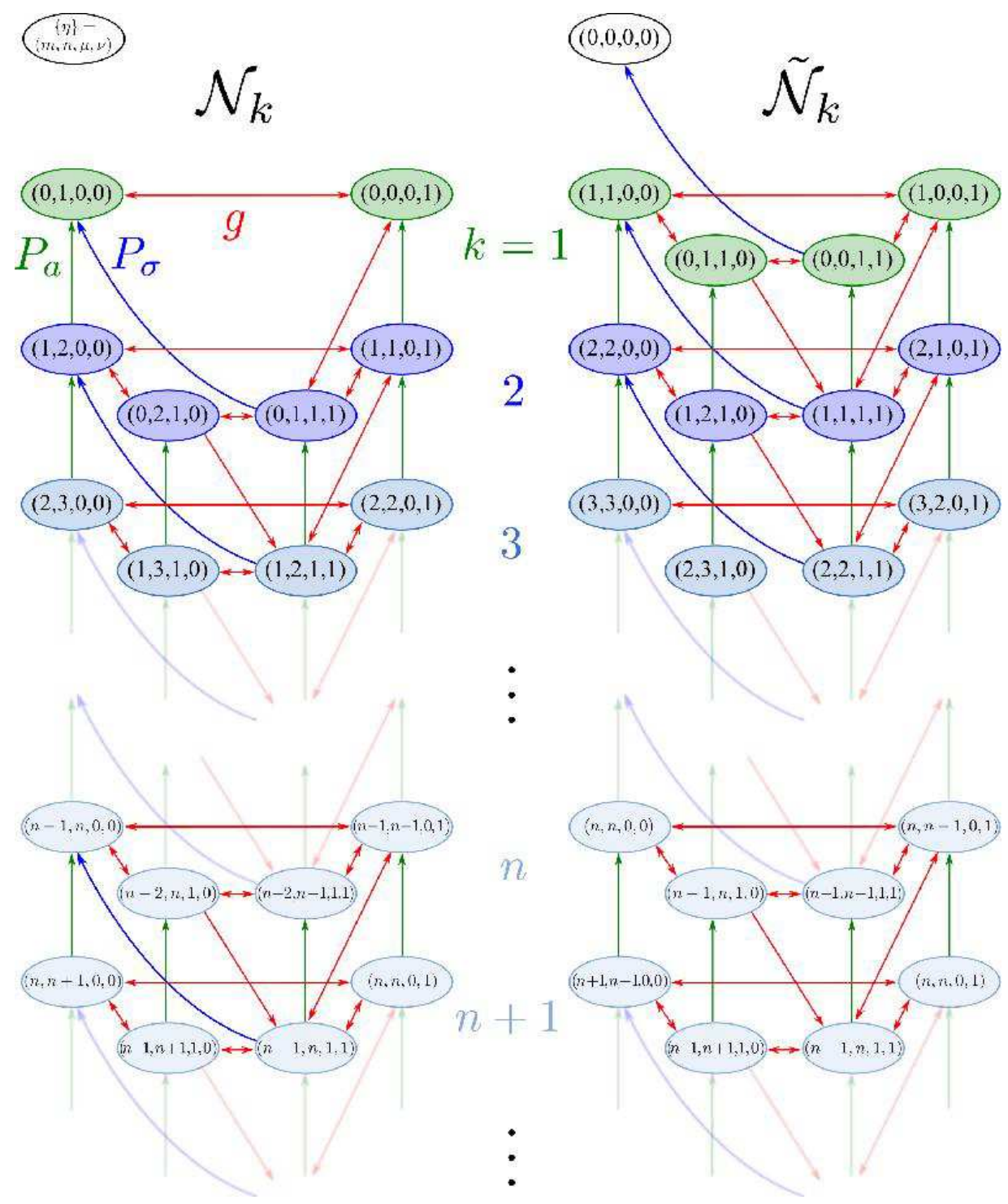

FIG. 1: (Color online) Chain of correlators - indexed by $\{\eta\}=(m, n, \mu, \nu)$-linked by the dissipative Jaynes-Cummings dynamics. On the left (resp., right), the set $\bigcup_{k} \mathcal{N}_{k}$ (resp., $\bigcup_{k} \tilde{\mathcal{N}}_{k}$ ) involved in the equations of the two-time (resp., single-time) correlators. In green are shown the first manifolds $\mathcal{N}_{1}$ and $\tilde{\mathcal{N}}_{1}$ that correspond to the Boson case, and in increasingly lighter shades of blues, the higher manifolds $\mathcal{N}_{k}$ and $\tilde{\mathcal{N}}_{k}$. The equation of motion $\left\langle a^{\dagger}(t) C_{\{\eta\}}(t+\tau)\right\rangle$ (resp. $\left\langle\sigma^{\dagger}(t) C_{\{\eta\}}(t+\tau)\right)$ with $\eta \in \mathcal{N}_{k}$ requires for its initial value the correlator $\left\langle C_{\{\tilde{\eta}\}}\right\rangle$ with $\{\tilde{\eta}\} \in \tilde{\mathcal{N}}_{k}$ defined from $\{\eta\}=(m, n, \mu, \nu)$ by $\{\tilde{\eta}\}=(m+1, n, \mu, \nu)$ (resp. $(m, n, \mu+1, \nu))$, as seen on the diagram. The red arrows indicate which elements are linked by the coherent (SC) dynamics, through the coupling strenght $g$, while the green/blue arrows show the connections due to the incoherent cavity/exciton pumpings, respectively. The self-coupling of each node to itself is not shown. This is where $\omega_{a, \sigma}$ and $\gamma_{a, \sigma}$ enter. 
and $D_{p}=[\mathbf{D}]_{p p}$ (when we refer to elements of a matrix or a vector by its indices, we enclose it with square brackets to distinguish from labelling indices). Further defining $\gamma_{p}$ and $\omega_{p}$ as the real and imaginary parts, respectively, of $D_{p}$

$$
\gamma_{p}+i \omega_{p}=D_{p}
$$

we can write Eq. (16) in a less concise but more transparent way. To all orders, it reads:

$$
S_{c}(\omega)=\frac{1}{\pi} \lim _{n_{\mathrm{t}} \rightarrow \infty} \sum_{p=1}^{s_{\mathrm{t}}}\left(L_{i_{c} p}^{c} \frac{\gamma_{p}}{\left(\omega-\omega_{p}\right)^{2}+\gamma_{p}^{2}}-K_{i_{c} p}^{c} \frac{\omega-\omega_{p}}{\left(\omega-\omega_{p}\right)^{2}+\gamma_{p}^{2}}\right) .
$$

Equation (19) brings together all the important quantities that define the luminescence spectrum of a quantum dot in a microcavity. The lineshape is composed of a series of Lorentzian and Dispersive parts, whose positions and broadenings are specified by $\omega_{p}$ and $2 \gamma_{p}$, cf. Eq. (18), and which are weighted by the coefficients $L_{i_{c} p}^{c}$ and $K_{i_{c} p}^{c}$, cf. Eq. (17). The former pertain to the structure of the spectral shape as inherited from the Jaynes-

Cummings energy levels. They are, as such, independent of the channel of detection (cavity or direct exciton emission). We devote Section [V] to them. The latter reflect the quantum state that has been realized in the system under the interplay of pumping and decay. They determine which lines actually appear in the spectra, and with which intensity. Naturally, the channel of emission is a crucial element in this case. We devote Section $\nabla$ to this aspect of the problem.

\section{SPECTRAL STRUCTURE}

In this Section, we discuss the series of coefficients $\omega_{p}$ and $\gamma_{p}$ that in the luminescence spectrum, Eq. (19), determine the position and the broadening (Half-Width at Half Maximum, HWHM) of the lines, respectively, be it the cavity or direct exciton emission. The case of vanishing pumping is fundamental, as it corresponds to the textbook Jaynes-Cummings results with the spontaneous emission of an initial state. It serves as the skeleton for the general case with arbitrary pumping and supports the general physical picture. Finally, it admits analytical results. We therefore begin with the case where $P_{a}, P_{\sigma} \ll \gamma_{a}, \gamma_{\sigma}$. The eigenvalues of the matrix of regression $\mathbf{M}$, are grouped into manifolds. There are two for the first manifold, given by:

$$
D_{\frac{1}{2}}=\Gamma_{+}+i\left(\omega_{a}-\frac{\Delta}{2} \mp \sqrt{g^{2}-\left(\Gamma_{-}+i \frac{\Delta}{2}\right)^{2}}\right),
$$



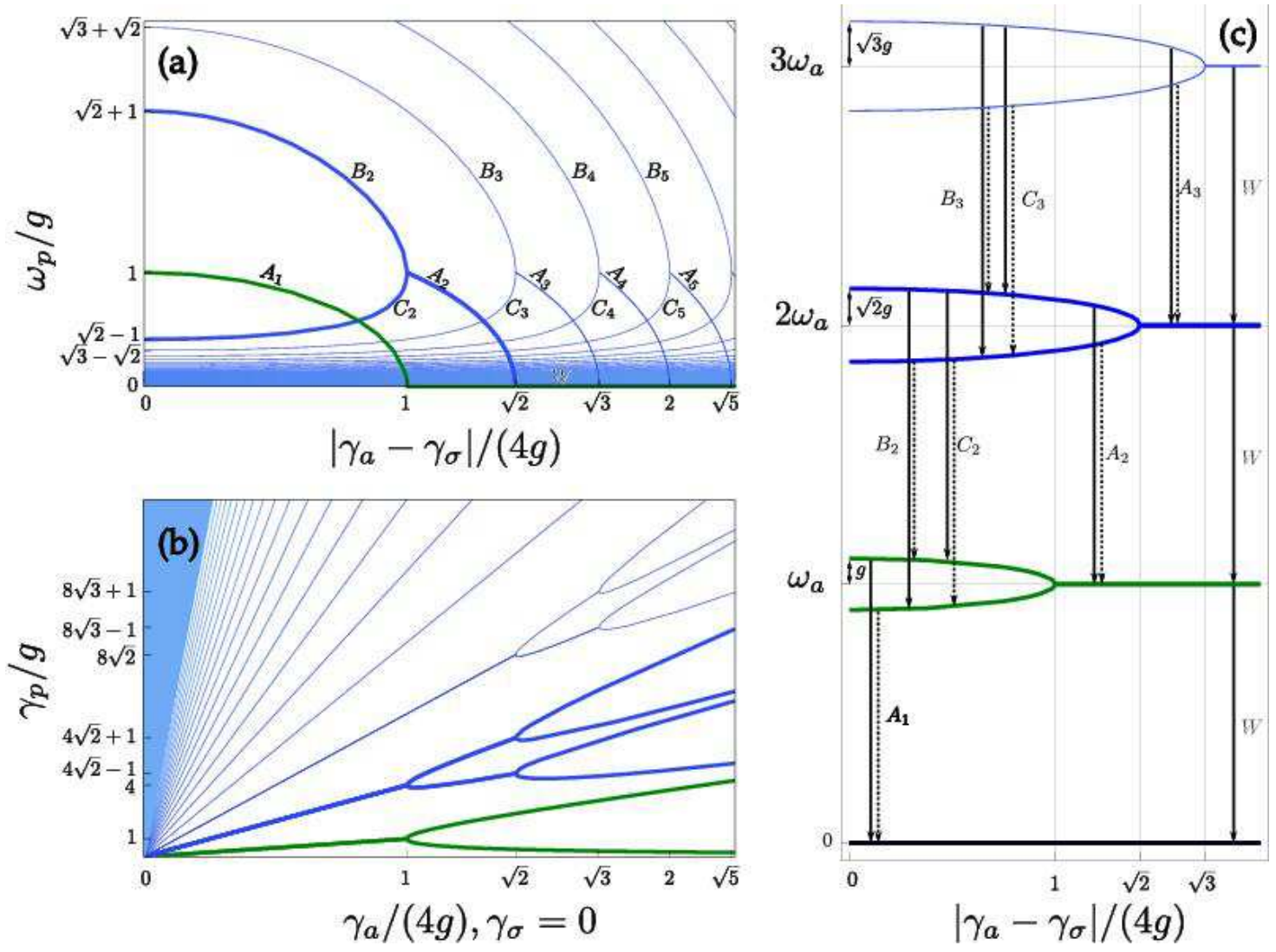

FIG. 2: (Color online) Spectral structure of the Jaynes-Cummings model at resonance and without pumping. (a) Positions $\omega_{p}$ of the lines in the luminescence spectrum. Only energies higher than $\omega_{a}$ are shown (not their symmetric below $\omega_{a}$ ). We take $\omega_{a}=0$ as the reference energy. In green (thick), the first manifold, and in increasing shades of blue, the successive higher manifolds which form a pattern of branch-coupling curves that define different orders of SC. (b) Half-Width at Half Maximum (HWHM) $\gamma_{p}$ of the lines (with $\gamma_{\sigma}=0$ ). In both (a) and (b), the blue filled region results from the accumulation of the countable-infinite vanishing lines. (c) Eigenenergies of the Jaynes-Cummings Hamiltonian with decay as an imaginary part of the bare energies (the JaynesCummings ladder). This provides a clear physical picture of panel (a) where the peaks positions arise from the difference of energy between lines of two successive manifolds. Lines $A_{k}$ of (a) stem from the emission from manifold $k$ in SC into manifold $k-1$ in WC (or vacuum). Lines $B_{k}$ and $C_{k}$ stem from the emission between the two manifolds in SC. Solid lines are those plotted in (a), dotted lines produce the symmetric lines, not shown. The horizontal line $W$ at 0 in (a) arises from decay between two manifolds in WC. Scheme (c) also reproduces the broadening of the lines (b) with the sum of the imaginary parts of the eigenenergies involved in the transition. 
and four for each manifold of higher order $k>1$, given by, for $4 k-5 \leq p \leq 4 k-2$ :

$$
D_{p}=\Gamma_{k}+i\left(\omega_{a}+\operatorname{sgn}(p-(8 k-7) / 2) R_{k}+(-1)^{p} R_{k-1}^{*}\right)
$$

$(\operatorname{sgn}(x)$ is defined as 0 for $x=0$ and $x /|x|$ otherwise), in terms of the $k$ th-manifold (half) Rabi splitting:

$$
R_{k}=\sqrt{(\sqrt{k} g)^{2}-\left(\Gamma_{-}+i \frac{\Delta}{2}\right)^{2}},
$$

and of the kth-manifold (half) broadening:

$$
\Gamma_{k}=(2 k-3) \Gamma_{-}+(2 k-1) \Gamma_{+}=(k-1) \gamma_{a}+\frac{\gamma_{\sigma}}{2}
$$

For each manifold, we have defined the $D_{p}$ in order by increasing value of the line position $\omega_{p}$.

According to Eq. (18), these provide the position $\omega_{p}$ of the line and its half-broadening $\gamma_{p}$ through their imaginary and real parts. $\Gamma_{k}$ is always real, so contributes in all cases to $\gamma_{p}$ only. $R_{k}$ is (at resonance) either pure real, or pure imaginary, and similarly to the boson case, this is what defines SC. This corresponds to an oscillatory or damped field dynamics of the two-time correlators within manifold $k$, which lead us to the formal definition: WC and SC of order $n$ are defined as the regime where the complex Rabi frequency at resonance, Eq. (22), is pure imaginary (WC) or real (SC). The criterion for $n$th order SC is therefore:

$$
g>\left|\Gamma_{-}\right| / \sqrt{n}
$$

$\mathrm{SC}$ is achieve more easily for given system parameters $\left(g\right.$ and $\left.\gamma_{a, \sigma}\right)$, with an increasing photon-field intensity that enhances the effective coupling strength. The lower the SC order, the stronger the coupling. This corresponds to the $n$th manifold (and all above) being in SC (aided by the cavity photons), while the $n-1$ manifolds below are in WC. First order is therefore the one where all manifolds are in SC. Equation (24) includes the SC in the standard boson case [1], $g>\left|\Gamma_{-}\right|$, as the first order SC of the fermion case, that is shown in green (thick) in Fig. 2. The same position of the peaks $\omega_{1,2}$ and the same (half) broadenings $\gamma_{1,2}$ is also recovered (in the absence of pumping). Note that similarly to the boson case, the SC is defined by a comparison between the coupling strenght $g$ with the difference of the effective broadening $\Gamma_{a}$ and $\Gamma_{b}$. The sum of these play no role in this regard.

The $\omega_{p}$ and $\gamma_{p}$ are plotted in Fig. 2(a) and 2(b), respectively, as function of $\Gamma_{-}$. Note that $\omega_{p}$ only depends on $g$ and $\Gamma_{-}$, whereas $\gamma_{p}$ also depends on $\Gamma_{+}$(that is why we plot it for $\left.\gamma_{\sigma}=0\right)$. 
The $D_{p}$, Eq. (21), have a natural interpretation in terms of transitions between the manifolds of the so-called Jaynes-Cummings ladder. The eigenenergies of the Jaynes-Cummings Hamiltonian with decay granted as the imaginary part of the bare energies $\left(\omega_{a, \sigma}-i \gamma_{a, \sigma} / 2\right)$, are given by $E_{ \pm}^{k}$ with

$$
E_{ \pm}^{k}=k \omega_{a}-\frac{\Delta}{2} \pm R_{k}-i \frac{(2 k-1) \gamma_{a}+\gamma_{b}}{4},
$$

for the $k$ th manifold. The four possible transitions between consecutive manifolds $k$ and $k-1$ give rise, when $k>1$, to the four peaks we found:

$$
\begin{array}{ll}
D_{4 k-5}=i\left[E_{-}^{k}-\left(E_{+}^{k-1}\right)^{*}\right], & D_{4 k-4}=i\left[E_{-}^{k}-\left(E_{-}^{k-1}\right)^{*}\right], \\
D_{4 k-3}=i\left[E_{+}^{k}-\left(E_{+}^{k-1}\right)^{*}\right], & D_{4 k-2}=i\left[E_{+}^{k}-\left(E_{-}^{k-1}\right)^{*}\right] .
\end{array}
$$

In the case $k=1$, only the two peaks common with the linear regime arise, $D_{1,2}=i E_{\mp}^{1}$, given respectively by Eqs. (26a) and (26b) with $E^{0}=0$. The fact that the $D_{p}$ correspond to $i\left[E^{k}-\left(E^{k-1}\right)^{*}\right]$ shows that, although the positions of the lines are given by a difference, their broadenings are given by a sum (because of complex conjugation). Physically, the uncertainties in the initial and final states indeed add up in the uncertainty of the transition energy.

The ladder is shown (at resonance) in Fig. 2(c). Let us discuss it in connection with our definition of SC in this system, to arbitrary $n$. When $\Gamma_{-}=0$, each step of the ladder is constituted by the two eigenstates of the Fermion dressed by the $n$ cavity photons, resulting in a splitting of $2 \sqrt{n} g$. This $n$-dependent splitting produces quadruplets of delta peaks with splitting of $\pm(\sqrt{n} \pm \sqrt{n-1}) g$ around $\omega_{a}$, as opposed to the boson case where independently of the manifold, the peaks are all placed at $\pm g$ around $\omega_{a}$. In a more general situation with $\Gamma_{-} \neq 0$, there are three possibilities for a manifold $k>1$ :

1. Both manifold $k$ and $k-1$ are in $S C$. The two Rabi coefficients $R_{k}$ and $R_{k-1}$ are real. This is the case when

$$
\left|\Gamma_{-}\right| \leq g \sqrt{k-1} .
$$

The luminescence spectra corresponds to four splitted lines $\omega_{p} \rightarrow \omega_{a} \pm\left(R_{k} \pm R_{k-1}\right)$, coming from the four possible transitions [Eqs. (26)), shown as $B_{k}$ and $C_{k}$ in Fig. 2(c)] between manifolds $k$ and $k-1$. The emission from all the higher manifolds also produces four lines. They are grouped pairwise around $\omega_{a}$ [Fig. 2(a)] and all have the same broadening, contributed by $\Gamma_{k}$ only [the single straight line in Fig. 2(b)]. 
2. Manifold $k$ is in $S C$ while manifold $k-1$ is in $W C$. In this case, $R_{k}$ is pure imaginary (contributing to line positions) and $R_{k-1}$ is real (contributing to broadenings). This is the case when

$$
g \sqrt{k-1}<\left|\Gamma_{-}\right|<g \sqrt{k} .
$$

This corresponds to two lines $\omega_{p} \rightarrow \omega_{a} \pm R_{k}$ in the luminescence spectrum, coming from the two possible transitions [shown as $A_{k}$ in Fig. 22(c)] between the SC manifold $k$ and the WC manifold $k-1$. Each of them is doubly degenerated. The two contributions at a given $\omega_{p}$ have two distinct broadenings $\gamma_{p} \rightarrow \Gamma_{k} \pm\left|R_{k-1}\right|$ around $\Gamma_{k}$. [cf. Fig. 2(b)]. The final lineshapes of the two lines $A_{2}$ is the same. In this region, all the emission from the higher manifolds produce four lines and all from the lower produce only one (at $\omega_{a}$ ), being in WC.

3. Both manifold $k$ and $k-1$ are in $W C$. The two Rabi coefficients $R_{k}$ and $R_{k-1}$ are pure imaginary. This is the case when

$$
g \sqrt{k} \leq\left|\Gamma_{-}\right| .
$$

This corresponds to only one line at $\omega_{p} \rightarrow \omega_{a}$ in the luminescence spectrum, coming from the transition from one manifold in WC to the other [shown as $W$ in Fig. 2(c)]. The line is four-time degenerated, with four contributions with different broadenings $\gamma_{p} \rightarrow \Gamma_{k} \pm\left(\left|R_{k}\right| \pm\left|R_{k-1}\right|\right)$, as seen in Fig. 2(b).

Figure 2 is the skeleton for the luminescence spectra - whether that of the cavity or of the direct exciton emission. It specifies at what energies can be the possible lines that constitutes the final lineshape, and what are their broadening. To compose the final result, we only require to know the weight of each of these lines.

In the $\mathrm{SE}$ case, the weights $L_{p}$ and $K_{p}$ include the integral of the single-time mean values $\mathbf{v}_{a}(t, t)$ over $0 \leq t<\infty$. Therefore, only those manifolds with a smaller number of excitations than the initial state can appear in the spectrum. Each of them, will be weighted by the specific dynamics of the system. The "spectral structure" - i.e., the $\omega_{p}$ and $\gamma_{p}$-depends only the system parameters $\left(g\right.$ and $\left.\gamma_{a, \sigma}\right)$. Therefore, in the SE case, the resulting emission spectrum is an exact mapping of the spectral structure of the Hamiltonian, Fig. 2.

In the SS case, the weighting of the lines also depends on which quantum state is realized, this time under the balance of pumping and decay. But the excitation scheme also changes 
the spectral structure of Fig. 2. When the pumping parameters are small, the changes will mainly be perturbations of the present picture and most concepts will still hold, such as the definition of SC, Eq. (24) for nonzero $P_{a, \sigma}$ in (11). However, when the pump parameters are comparable to the decay parameters, the manifold picture in terms of Hamiltonian eigenenergies breaks. The underlying spectral structure must be computed numerically for each specific probing of the system with $P_{a}$ and $P_{\sigma}$. It can still be possible to identify the origin of the lines with the manifold transitions by plotting their position $\omega_{p}$ as a function of the pumps, starting from the analytic limit. SC of each manifold can be associated to the existence of peaks positioned at $\omega_{p} \neq \omega_{a}, \omega_{\sigma}$. We address this problem in next Sections.

\section{POPULATION AND STATISTICS}

To know which features of the spectral structure dominate and which are negligible, one needs to know what is the quantum state of the system. In the boson case, it was enough to know the average photon $\left(n_{a}\right)$ and exciton $\left(n_{b}\right)$ numbers, and the off-diagonal element $n_{a b}=\left\langle a^{\dagger} b\right\rangle$. In the most general case of the fermion system, a countably infinite number of parameters are required for the exact lineshape. The new order of complexity brought by the fermion system is illustrated for even the simplest observable. Instead of a closed relationship that provides, e.g., the populations in terms of the system parameters and pumping rates, only relations between observables can be obtained in the general case. For instance, for the populations:

$$
\Gamma_{a} n_{a}+\Gamma_{\sigma} n_{\sigma}=P_{a}+P_{\sigma}
$$

This expression is formally the same as for the coupling of two bosonic modes. The differences are in the effective dissipation parameter $\Gamma_{\sigma}=\gamma_{\sigma}+P_{\sigma}$ (instead of the bosonic one, $\left.\gamma_{b}-P_{b}\right)$ and the constrain of the exciton population, $0 \leq n_{\sigma} \leq 1$. One solution of Eq. (30) is $n_{a}^{\text {th }}=P_{a} / \Gamma_{a}$ and $n_{\sigma}^{\text {th }}=P_{\sigma} / \Gamma_{\sigma}$, which corresponds to the case $g=0$, where each mode reaches its thermal steady state independently (Bose/Fermi distributions, depending on the mode statistics). With coupling $g \neq 0$, we can only derive some analytical limits and bounds. For example, when $\gamma_{a}=P_{a}$, one sees from Eq. (30) that $n_{\sigma}=\left(P_{\sigma}+P_{a}\right) / \Gamma_{\sigma}$, with the condition for the cavity pump $P_{a} \leq \gamma_{\sigma}$ (since $\left.n_{\sigma} \leq 1\right)$. If only the dot is pumped, $n_{\sigma}=n_{\sigma}^{\text {th }}$, and if both $P_{a}, \gamma_{a}=0$ then, also $n_{a}=n_{a}^{\text {th }}=P_{\sigma} /\left(\gamma_{\sigma}-P_{\sigma}\right)$ with the same tempera- 
ture. As, in this case, $P_{\sigma}$ must be strictly smaller than $\gamma_{\sigma}$, the exciton population $n_{\sigma} \leq 1 / 2$ prevents an inversion of population, as is well known for a two-level system.

When $\gamma_{a}>P_{a}$, we get the following bounds for the cavity populations in terms of the system and pumping parameters:

$$
\frac{P_{a}-\gamma_{\sigma}}{\gamma_{a}-P_{a}} \leq n_{a} \leq \frac{P_{a}+P_{\sigma}}{\gamma_{a}-P_{a}} .
$$

When $P_{\sigma}=\gamma_{\sigma}=0$, the cavity is in thermal equilibrium with its bath, $n_{a}=n_{a}^{\text {th }}$, and with the $\operatorname{dot} n_{\sigma}=P_{a} /\left(\gamma_{a}+P_{a}\right)$. In this case, the pump is limited by $P_{a}<\gamma_{a}$, and again $n_{\sigma} \leq 1 / 2$. Again, the inversion of population cannot take place putting the system in contact with only one thermal bath. In all these situations where an analytic expression for the population is obtained, the detuning between cavity and dot does not affect the final steady state, although it determines, together with the coupling strength, the time that it takes to reach it. An interesting limiting case where inversion can happen, is that where $\gamma_{\sigma}$ and $P_{a}$ are negligible, then $n_{a}=P_{\sigma}\left(1-n_{\sigma}\right) / \gamma_{a}$. When the pump is low and $n_{\sigma}<1, n_{a}$ grows with pumping, but when the dot starts to saturate and $n_{\sigma} \rightarrow 1$ the cavity population starts to quench towards $n_{a} \rightarrow 0$ [36]. Here, all values of $P_{\sigma}$ bring the system into a steady state as $n_{a}$ cannot diverge. However, if we allow some cavity pumping, given that $a$ does not saturate, $P_{a}$ is bounded. A rough guess of this boundary is, in the most general case:

$$
P_{a}<\max \left(\gamma_{a}, \gamma_{\sigma}\right) .
$$

If Eq. (32) is not fulfilled, the system diverges, as more particles are injected at all times by the incoherent cavity pumping than are lost by decay. Numerical evidence suggests that the actual maximum value of $P_{a}$ depends on $P_{\sigma}$. To some given order $n_{t}$, divergence typically arises much before condition (32) is reached, although it is difficult to know if a lower physical limit has been reached or if the order of truncation was not high enough.

The second order correlator $g^{(2)}$ can be expressed as a function of $n_{a}$ only:

$$
\begin{aligned}
g^{(2)}=\left[g ^ { 2 } \left(\left(n_{a}+1\right)\left(P_{a}^{2}+P_{\sigma}^{2}\right)-n_{a}\left(\gamma_{a}+\gamma_{\sigma}\right)^{2}+P_{a}\left(\gamma_{a}+\Gamma_{\sigma}+P_{\sigma}+6 n_{a} \Gamma_{\sigma}\right)\right.\right. \\
\left.\left.\quad+P_{\sigma}\left(\gamma_{a}+\gamma_{\sigma}-2 n_{a} \gamma_{a}\right)\right)+\left(P_{a}-n_{a} \Gamma_{a}\right) \Gamma_{\sigma}\left(4 \Gamma_{+}^{2}+\Delta^{2}\right)\right] / 2 g^{2} n_{a}^{2} \Gamma_{a} \Gamma_{\sigma} .
\end{aligned}
$$

Obtaining the expression for the $n$th order correlator and setting it to zero would provide an approximate (of order $n$ ) closed relation for $n_{a}$. We shall not pursue this line of research that becomes very heavy. 


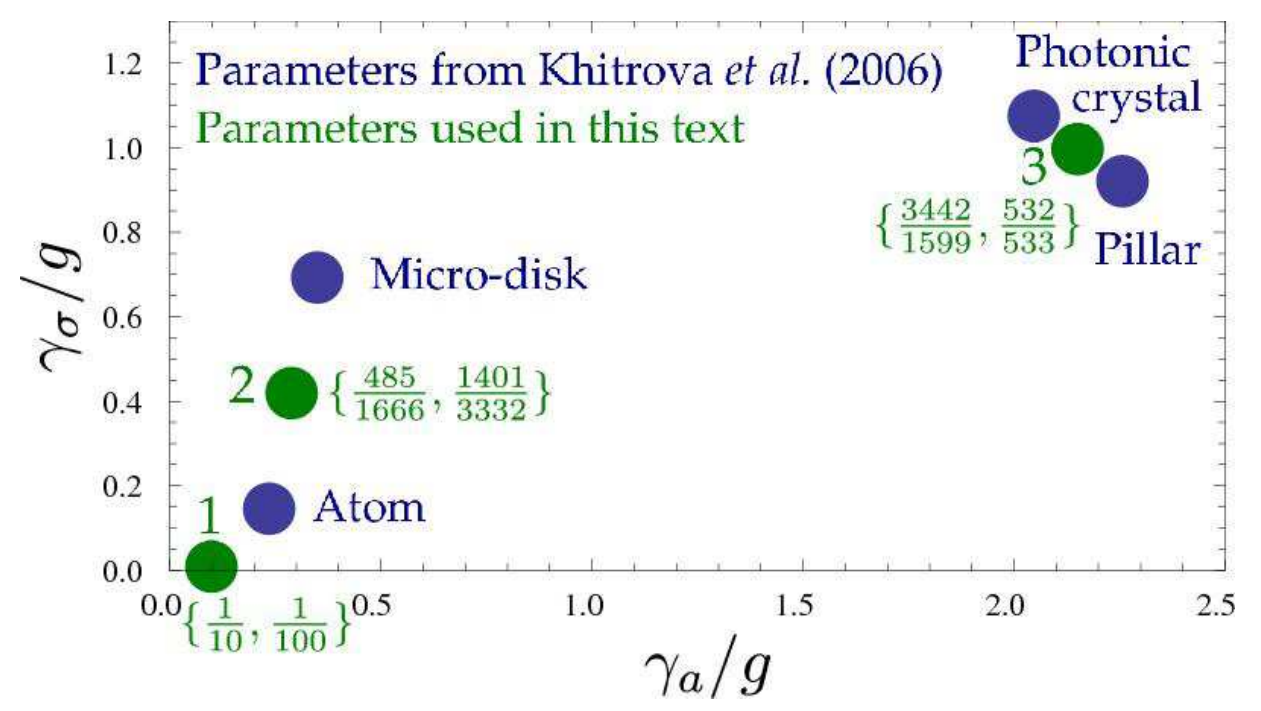

FIG. 3: (Colour online) Blue points give the decay rates for the cavity and quantum dot estimated by Khitrova et al. in Ref. [34] for four references systems having achieved SC at this time: photonic crystals and pillar microcavities nearby point 3, microdisks and atomic systems nearby point 2 . In Green, the three sets of parameters used in this text. Points 2 and 3 average over their two nearest neighbors and represent these systems. Point 1 represents a very good system in very strong coupling, that might be realizable in the near future. Parameters are fractions because numerical computations have been done to arbitrary precisions (with the values given).

As an overall representation of the typical systems that arise in real and desired experiments, we consider three configurations, shown in Fig. (3), scattered in order to give a rough representative picture of the overall possibilities, around parameters estimated in Ref. [34]. Point 1 corresponds to the best system of our selection, in the sense that its decay rates are very small $\left(\gamma_{a}=g / 10, \gamma_{\sigma}=g / 100\right)$, and the quantum (Hamiltonian) dynamics dominates largely the system. It is a system still outside of the experimental reach. Point 3 on the other hand corresponds to a cavity with important dissipations, that, following our analysis below, precludes the observation of any neat structure attributable to the underlying Fermi statistics. According to numerical fitting of the experiment, real structures might even be suffering higher dissipation rates [35]. Point 2 represents other lead systems of the $\mathrm{SC}$ physics, that we will show can presents strong departure from the linear regime, in particular conditions that we will emphasize. The best semiconductor system from Fig. 3 is realized with microdisks, thanks to the exceedingly good cavity factors. We shall not enter 

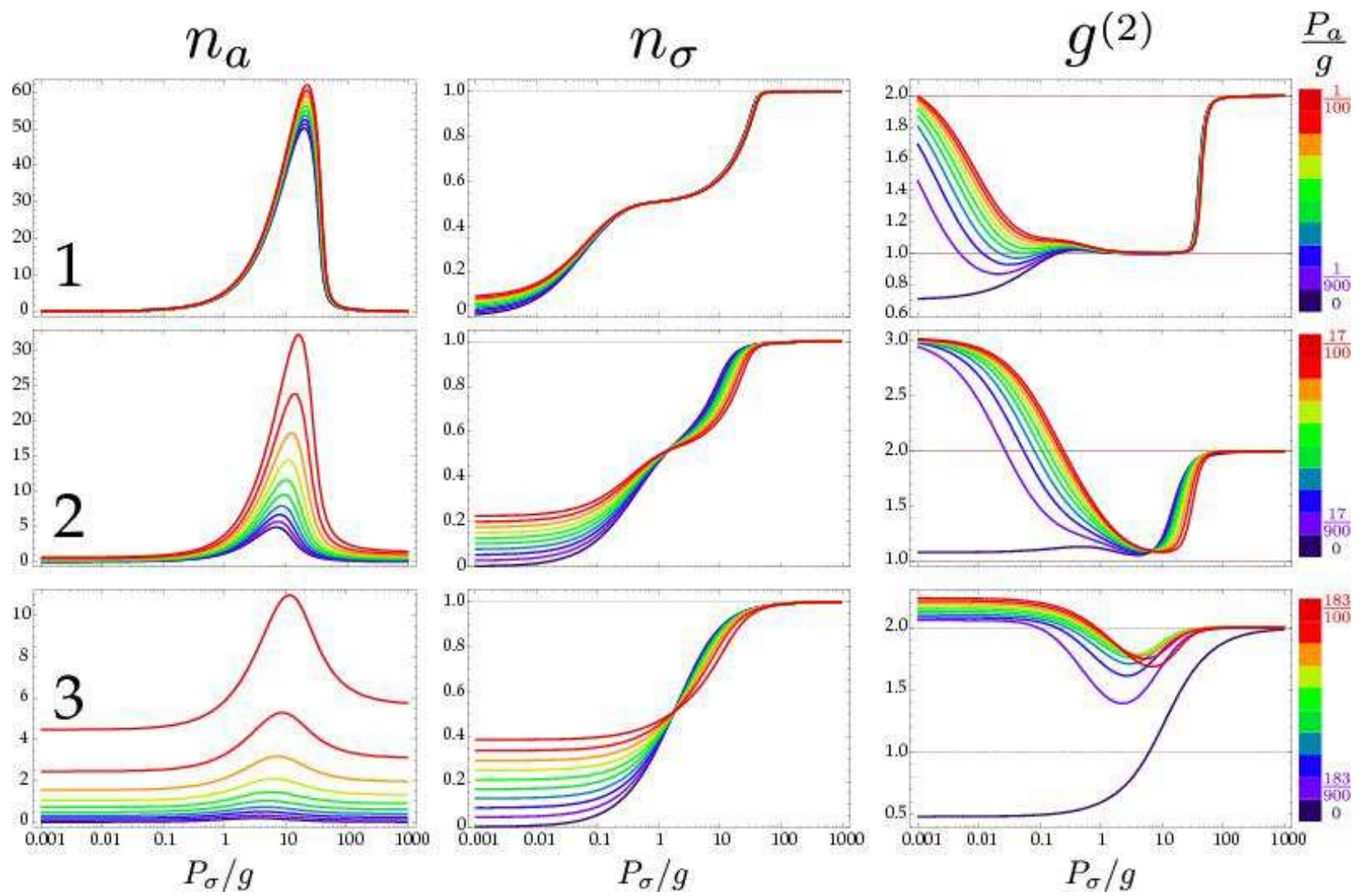

FIG. 4: Populations and statistics of the points marked 1,2 and 3 in Fig. 3, Each row shows the triplet $n_{a}$ (1st column), $n_{\sigma}(2 \mathrm{nd})$ and $g^{(2)}(0)(3 \mathrm{rd})$ for a given point ( $n$th row corresponds to point $n$ ). All plots share the same $x$-axis in log-scale of $P_{\sigma} / g$ ranging from $10^{-3}$ to $10^{3}$. All $y$-axis are rescaled to its specific graph, at the exception of $n_{\sigma}$ which is always between 0 and 1 . The color code corresponds to different values of $P_{a}$. Each color code applies to its row and is given in the last column. The qualitative behavior is roughly the same for all points: there is a peak in $n_{a}$ that is subsequently quenched as the dot gets saturated. In $g^{(2)}$, there is on the other hand, a local minimum of fluctuations that can be brought to the Poissonian limit of 1 (allowing for a lasing region) and maintained over a large plateau in good cavities.

into specific discussion of the advantages and inconvenient of the respective realizations and the accuracy of these estimations. From now on, we shall refer to this set of parameters as that of "reference points", keeping in mind that points 1 and 2 in particular represent systems that we will refer to as a "good system" and a "more realistic system", respectively.

In Fig. 4, the three observable of main interest for a physical understanding of the system that we have just discussed $-n_{a}, n_{\sigma}$ and $g^{(2)}$-are obtained numerically for the three reference 
points. Electronic pumping is varied from, for all practical purposes, vanishing $\left(10^{-3} \mathrm{~g}\right)$ to infinite $\left(10^{3} \mathrm{~g}\right)$ values. Various cavity pumpings are investigated and represented by the color code from no-cavity pumping (dark blue) to high, near diverging, cavity pumping (red), through the color spectrum. We checked numerically that these results satisfy Eq. (30). The overall behavior is mainly known, for instance the characteristic increase till a maximum and subsequent decrease of $n_{a}$ with $P_{\sigma}$ has been predicted in a system of QD coupled to a microsphere [36]. This phenomenon of so-called self-quenching is due to the excitation impairing the coherent coupling of the dot with the cavity: bringing in an exciton too early disrupts the interaction between the exciton-photon pair formed from the previous exciton. Therefore the pumping rate should not overcome significantly the coherent dynamics. Too high electronic pumping forces the QD to remain in its excited state and thereby prevents it from populating the cavity. In this case the cavity population returns to zero while the exciton population (or probability for the QD to be excited) is forced to one. The cavity pumping brings an interesting extension to this mechanism. First there is no quenching for the pumping of bosons that, on the contrary, have a natural tendency to accumulate and lead to a divergence. Therefore the limiting values for $n_{a}$ when $P_{\sigma} \rightarrow 0$ or $P_{\sigma} \rightarrow \infty$ are not zero, as in the previously reported self-quenching scenario [36]. They also happen to be different:

$$
\begin{aligned}
& n_{a}^{<} \equiv n_{a}\left(P_{\sigma}=0\right)=\frac{P_{a}-\gamma_{\sigma} n_{\sigma}}{\gamma_{a}-P_{a}}, \\
& n_{a}^{>} \equiv \lim _{P_{\sigma} \rightarrow \infty} n_{a}=\frac{P_{a}}{\gamma_{a}-P_{a}},
\end{aligned}
$$

and therefore satisfy $n_{a}^{<}<n_{a}^{>}$. Eq. (34b) follows from the decoupled thermal values for the populations, $n_{\sigma} \rightarrow P_{\sigma} /\left(P_{\sigma}+\gamma_{\sigma}\right)$, and corresponds to a passive cavity where the quenched dot does not contribute at all. In this case, the emission spectrum of the system is expected to converge to

$$
S_{a}(\omega)=\frac{1}{\pi} \frac{\Gamma_{a} / 2}{\left(\omega-\omega_{a}\right)^{2}+\left(\Gamma_{a} / 2\right)^{2}},
$$

for the cavity, and $S_{\sigma}(\omega)=0$ for the dot. The other limit when $P_{\sigma}=0$, shows the deleterious effect of the dot on cavity population. The dot fully enters the dynamics contrary to the quenched case where it is subtracted from it.

Important application of SC for single-atom lasing are to be found in good cavities 1 and 2, where the coupling $g \gg \gamma_{a}, \gamma_{b}$ is strong enough [37, 38, 39, 40, 41]. Lasing can occur 
when the pumping is also large enough to overcome the total losses, $P_{\sigma} \gg \gamma_{a}, \gamma_{\sigma}$. Setting $P_{a}$, $\gamma_{\sigma}=0$, Eqs. (66) can be approximately reduced to one for the total probability $\mathrm{p}[n]$ [36, 42]:

$$
\partial_{t} \mathrm{p}[n]=\gamma_{a}(n+1) \mathrm{p}[n+1]-\left(\gamma_{a} n+\frac{l_{G}(n+1)}{1+l_{S} / l_{G}(n+1)}\right) \mathrm{p}[n]+\frac{l_{G} n}{1+l_{S} / l_{G} n} \mathrm{p}[n-1]
$$

The parameters that characterize the laser are the gain $l_{G}=4 g^{2} / P_{\sigma}$ and the self saturation

$l_{S}=8 g^{2} l_{G} / P_{\sigma}^{2}$. Far above threshold $\left(n_{a} l_{S} / l_{G} \gg 1\right)$, the statistics are Poissonian, $g^{(2)}=1$, with a large intensity in the emission, $n_{a}=P_{\sigma} /\left(2 \gamma_{a}\right)$, and half filling of the dot, $n_{\sigma}=0.5$. However, this analytic limit from the standard laser theory is not able to reproduce the selfquenching effect induced by the incoherent pump, nor the subpoissonian region $\left(g^{(2)}<1\right)$ where quantum effects are prone to appear. The validity of the laser theory is restricted to the narrow region, $\gamma_{a} \ll P_{\sigma} \ll \gamma_{\sigma}^{\mathrm{P}}$, where $\gamma_{\sigma}^{\mathrm{P}}=4 g^{2} / \gamma_{a}$ is the boundary for the self-quenching. In the weak coupling regime, $\gamma_{\sigma}^{\mathrm{P}}$ is the well known Purcell enhanced spontaneous decay rate of an exciton through the cavity mode. In the strongly coupled system, it can be similarly understood as the rate at which an exciton transforms into a photon. If the excitons are injected at a higher rate, there is no time for such a coherent exchange to take place and populate the cavity with photons. Fig. 4 shows that lasing can be achieved with system 1 in the corresponding region of pump. Here, we will solve the system exactly, covering this regime and all the other possible ones with the full quantum equations of motion.

The effect of cavity pumping depends strongly on the experimental situation. In the case of an exceedingly good system, $P_{a}$ has little effect as soon as the exciton pumping is important, $P_{\sigma}>\gamma_{a}$. Cavity pumping becomes important again in a system like 2, where it enhances significantly the output power, with the price of superpoissonian statistics $\left(g^{(2)}>\right.$ 1). With a poorer system like point 3, some lasing effect can be found with the aid of the cavity pump: there is a nonlinear increase of $n_{a}$ and $g^{(2)}$ approaches 1 for $g<P_{\sigma}<10 g$. However, the weaker the coupling, the weaker this effect until it disappears completely for decay rates outside the range plotted in Fig. 3. In all cases, the self-quenching leads finally to a thermal mixture of photons $\left(g^{(2)}=2\right)$ and WC at large pumping.

\section{WEIGHTS AND RENORMALIZATION}

To give a complete picture of the spectral structure, that we have obtained analytically in Section IV] we need to consider how this limiting case of vanishing pumpings evolves with 

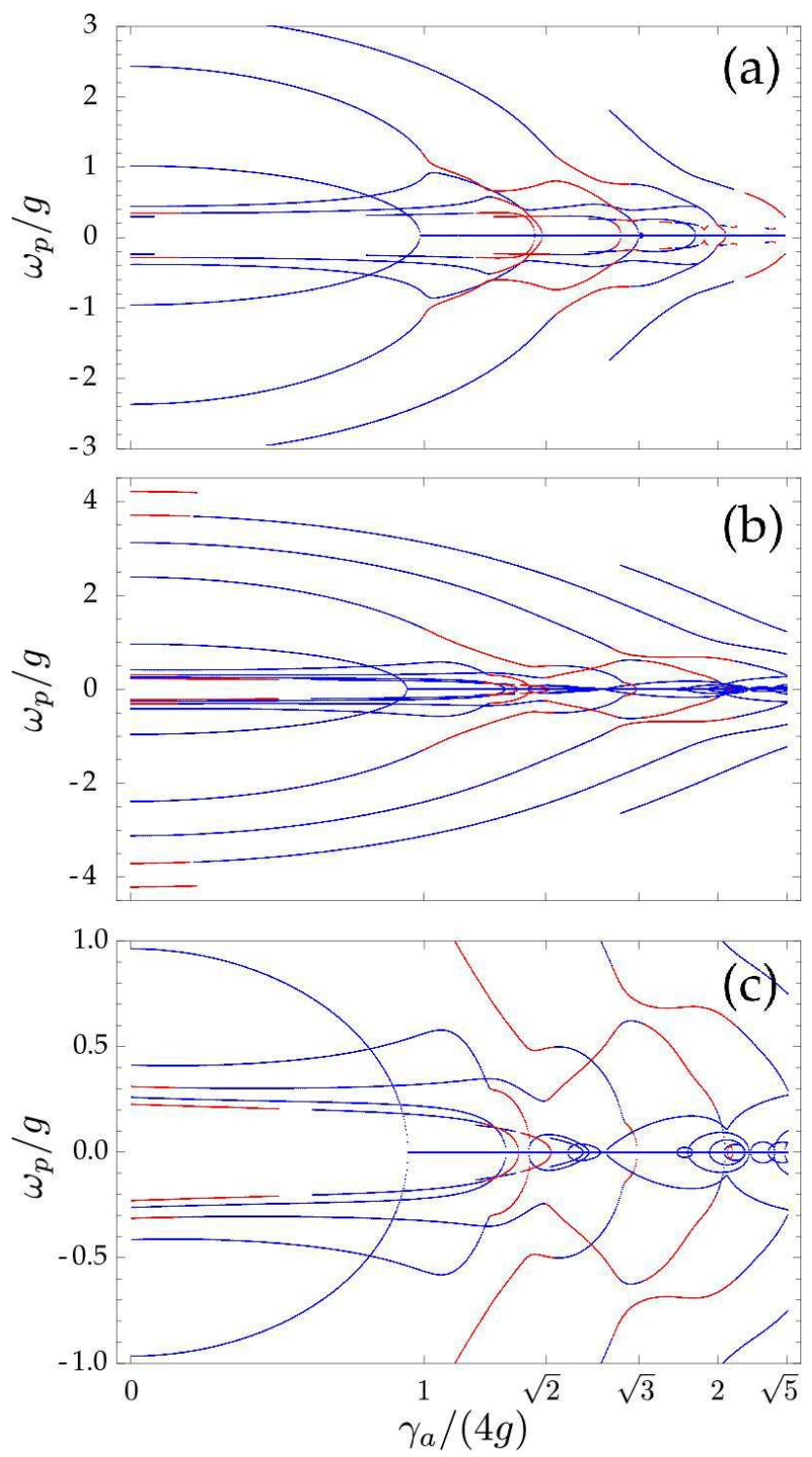

FIG. 5: (Color online) Spectral structure in the cavity emission of the Jaynes-Cummings model as a function of $\gamma_{a} / g$, with some electronic pumping $\left(\Delta=0, \gamma_{\sigma}=0, P_{a}=0, \omega_{a}=0\right)$. Panel (a) is for $P_{\sigma}=g / 50$ and (b)-(c) for $P_{\sigma}=g / 10$. Panel (c) is a zoom on the central peaks of the entire picture (b). In blue (resp., red) are the peaks with $L_{p}^{a}>0$ (resp., $L_{p}^{a}<0$ ).

finite pumping. Here again, we have to turn to numerical results.

Two cases of finite pumpings are shown in Fig. 5 for the finite pumping counterpart of Fig. 2(a), namely $P_{\sigma}=g / 50,(\mathrm{a})$, and $P_{\sigma}=g / 10,(\mathrm{~b})$ and (c). We take $\omega_{a}=0$ as the reference energy for the remaining of this text. Panel (a) shows how the limiting case $\left(P_{\sigma} \ll g\right)$ is weighted and deviates rather lightly from the analytical result. The computation has been made to truncation order $n_{t}=50$ and we checked that it had converged with other 


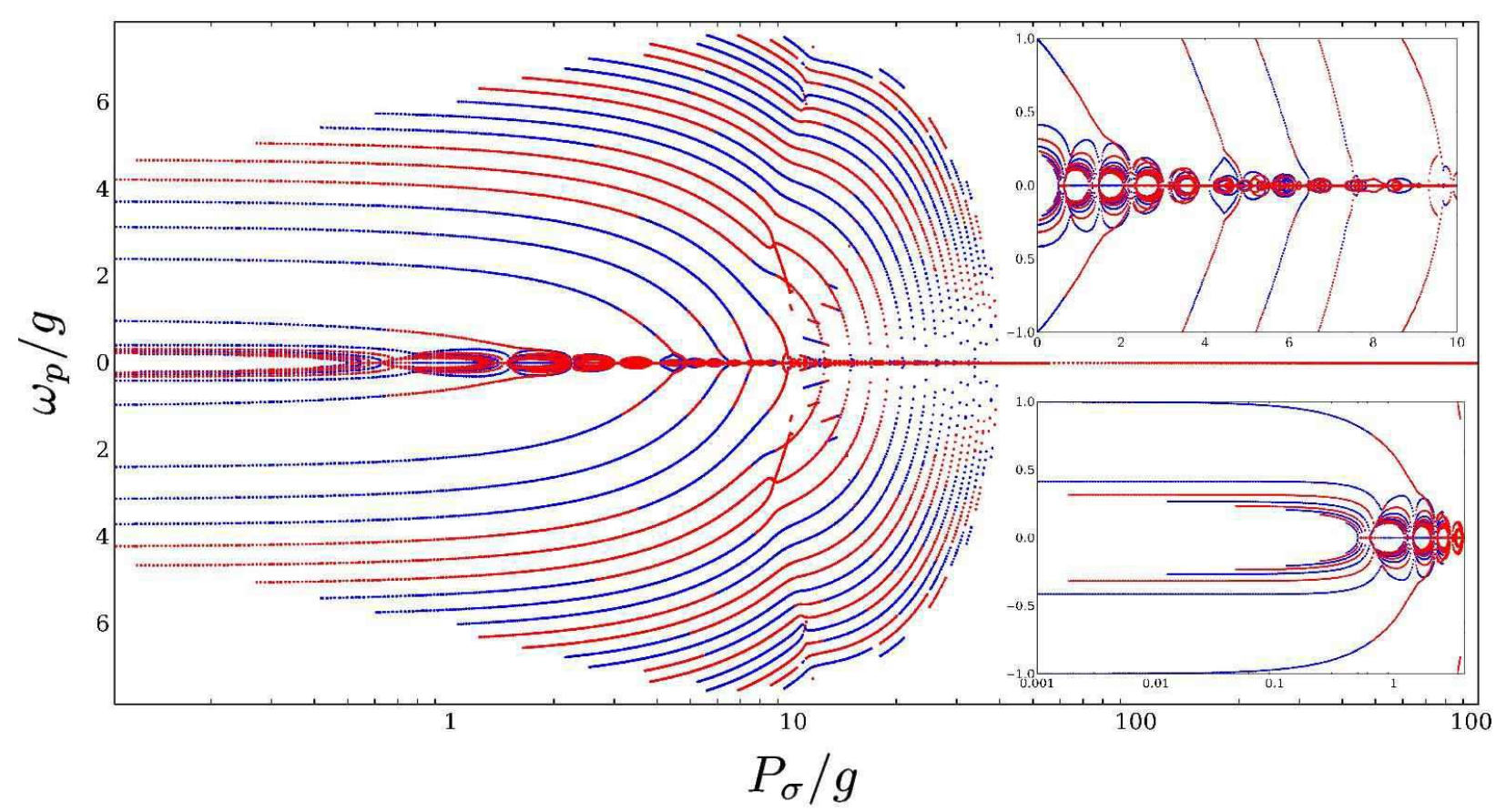

FIG. 6: (Color online) Spectral structure in the cavity emission at resonance as a function of $P_{\sigma} / g$ for Point $2\left(\Delta=0, P_{a}=0, \omega_{a}=0\right)$. In insets, we zoom over the central peaks and the region $0 \leq P_{\sigma} \leq g$ (upper) and $0 \leq P_{\sigma} \leq 10 g$ (lower), showing the complex structures that arise. In blue (resp., red) are the peaks with $L_{p}^{a}>0$ (resp., $\left.L_{p}^{a}<0\right)$. At sufficiently high pumping, all eigenvalues have collapse to zero, defining an extreme case of weak coupling.

truncation orders giving exactly the same result. In the figure, only $\omega_{p}$ whose weighting in the cavity emission $L_{p}^{a}$ (Lorentzian part) is nonzero are shown, although most of them are very small. If we plot only those with $\left|L_{p}^{a}\right| \geq 0.01$, only the usual vacuum Rabi doublet (in green in Fig. 2) would remain. In addition of the weight, also the degeneracy (number of peaks) at a given resonance should be taken into account to quantify the intensity of emission at a particular energy. This information is not apparent in the figures, where we only show in Blue or Red the cases of positive or negative, respectively, weighting. In some cases, many peaks superimpose with opposite signs, possibly cancelling each other. We plot negative values last so that a blue line corresponds to a region of only positive values, while a red line may come on top of a blue line. This figure gives nevertheless an insightful image of the underlying energy structure and how they contribute to the final spectrum as an addition of many emitting (or interfering) events. In (b) we show a case of higher pumping, 
with the same principal information to be found in the mapping of the eigenvalues. The characteristic branch-coupling of the Jaynes-Cummings, still easily identifiable in Fig. 5(a), has vanished, and lines of external peaks directly collapse toward the center. A zoom of the central part, panel (c), shows the considerable complexity of the inner peaks, forming "bubbles" around the central line, due to intensity-aided SC fighting against increasing dissipation that ultimately overtakes.

The origin of the lines can be better understood if we plot them as a function of pumping, as we commented in Section IV] In Fig. 6, the same weighted peak positions $\omega_{p}$ are shown

(with the same color code) for Point 2 as electronic pumping is varied from $10^{-3} \mathrm{~g}$ to $10^{3} \mathrm{~g}$ $\left(P_{a}=0\right)$. This last picture supports the idea that quantum effects (such as subpoissonian statistics, Fig. (4) are observed at small pumpings, with optimal range being roughly $P_{\sigma}<$ $0.5 \mathrm{~g}$, where only the lowest manifolds are probed. This is the range of pumping where the Jaynes-Cummings manifold structure is still close to that without pump. Further pumping pushes the lines to collapse, starting by the vacuum Rabi splitting which closes, evidencing the loss of the first order SC at $P_{\sigma} \approx 4 g$. Here again we observe this phenomenon of bubbling, with a sequence of lines opening and collapsing, that makes it impossible to specify the exact pump at which the transition takes place. From this point, SC is lost manifold by manifold similarly as in the case where $\gamma_{a}$ was increased. When $P_{\sigma} \approx 40 \mathrm{~g}$, all lines have collapsed onto the center and will remain so at higher pumpings. The dot saturates and the cavity empties with thermal photons in a WC regime.

In these conditions, either from Fig. 5(b)-(c) or Fig. 6, a general definition of StrongCoupling in presence of pumping is obviously very complex and remains to be established.

\section{LUMINESCENCE SPECTRA AT RESONANCE}

Now we have all the ingredients to present the final result: the spectral shapes of the system in a broad range of configurations and parameters. We cannot give a comprehensive picture as any set of parameters is by itself unique, but will instead illustrate the main trends, using specifically for that purposes the three reference points of Fig. 3. They give a good account of the general case and one can extrapolate from these particular cases how another configuration will behave. To get exact results for a given point, numerical computations must be undertaken. 
From now on, we shall represent in Blue the spectra that correspond to cavity emission and in Violet those that correspond to direct exciton emission. The main conclusions, based on semi-analytical results, are different for different points or family of points. Point 1, that represents a very good system, is the one that is best suited to explore quantum effects. Its spectral shape is unambiguously evidencing transitions in the Jaynes-Cummings ladder, as shown in Fig. [7 with a clear "Jaynes-Cummings fork" (a quadruplet). The outer peaks at \pm 1 are the conventional vacuum Rabi doublet, whereas the two inner peaks correspond to higher transitions in the ladder. Observation of a transition from outer to inner peaks with pumping such as shown in Fig. 7 would be a compelling evidence of a quantum exciton in SC with the cavity. Fig. 8 shows another multiplet structure of this kind for Point 1. The intensity of emission is presented in log-scale and for a broader range of frequencies, so that small features can be revealed. Transitions from up to the third manifold can be explicitly identified. The decay from the second manifold, that manifests distinctly with peaks labelled 2 (although it also contributes to peaks labelled 0), is already weak but still might be identifiable in an experimental PL measurement. Higher transitions have decreasing strenght. This can be checked with the probability to have $n$ photons in the cavity, $\mathrm{p}(n)$, computed from Eqs. (6). Whenever the mean number $n_{a}$ is low (as is the case here), this probability is maximum for the vacuum $(\mathrm{p}(n)>\mathrm{p}(n+1)$ for all $n)$, independently of the nature of the photon distribution (sub, super or Poissonian). Only when $n_{a}=1$, in the best of cases (for a Poissonian distribution), does this trend start to invert and $\mathrm{p}(1)=\mathrm{p}(0)$. This makes it impossible, even in the very good system of Point 1, to probe clearly and independently transitions between manifolds higher than 3, as their weak two outer peaks (approximately at $\pm(\sqrt{n}+\sqrt{n-1})$ ) are completely hidden by the broadening. A stronger manifestation of nonlinear emission is to be found in the pool of pairs of inner peaks from all high-manifold transitions (labelled 0 in Fig. 9), at approximately $\pm(\sqrt{n}-\sqrt{n-1})$. Not only the inner peaks coming from different manifolds are close enough to sum up, but also they are more intense than their outer counterparts. This can be easily understood by looking at the probability, $\mathrm{I}_{c}$, of transition between eigenstates $| \pm, n\rangle$ through the emission of a photon, $c=a$, or an exciton, $c=\sigma$. This probability, $\mathrm{I}_{c}^{(i \rightarrow f)} \propto|\langle f|c| i\rangle|^{2}$, estimates the relative intensity of the peaks depending on the initial, $|i\rangle$, and final, $|f\rangle$, states of the transition and on the channel of emission, $c=a, \sigma[3]$. A discussion in terms of the eigenstates of the Hamiltonian is still valid in the regime of Point 1 (very good system) at very low 
pump. At resonance, neglecting pumps and decays, the eigenstates for manifold $n$, are $|n, \pm\rangle=(|n, 0\rangle \pm|n-1,1\rangle) / \sqrt{2}$. The outer peaks arise from transitions between eigenstates of different kind, $|n, \pm\rangle \rightarrow|n-1, \mp\rangle$, while the inner peaks arise from transitions between eigenstates of the same kind, $|n, \pm\rangle \rightarrow|n-1, \pm\rangle$. Their probability amplitudes in the cavity emission,

$$
\begin{aligned}
& \mathrm{I}_{a}^{( \pm \rightarrow \mp)} \propto|\langle n-1, \mp|a| n, \pm\rangle|^{2}=|\sqrt{n}-\sqrt{n-1}|^{2} / 4 \\
& \mathrm{I}_{a}^{( \pm \rightarrow \pm)} \propto|\langle n-1, \pm|a| n, \pm\rangle|^{2}=|\sqrt{n}+\sqrt{n-1}|^{2} / 4
\end{aligned}
$$

evidence the predominance of the inner peaks versus the outer ones, given that one expect the same weighting of both transitions from the dynamics of the system. The doublet formed by the inner peaks is therefore strong and clearly identifiable in an experiment. On the other hand, in the direct exciton emission, the counterparts of Eqs. (37) are manifold-independent and equal for both the inner and outer peaks:

$$
\begin{aligned}
& \mathrm{I}_{\sigma}^{( \pm \rightarrow \mp)} \propto|\langle n-1, \mp|\sigma| n, \pm\rangle|^{2}=1 / 4, \\
& \mathrm{I}_{\sigma}^{( \pm \rightarrow \pm)} \propto|\langle n-1, \pm|\sigma| n, \pm\rangle|^{2}=1 / 4 .
\end{aligned}
$$

In this case, therefore, one can expect similar strength of transitions for both the inner and outer peaks with a richer multiplet structure for the direct exciton emission.

In Fig. 9, we give an overview of the PL spectra as $P_{\sigma}$ is varied from very small to very large values. For point 1, as we already noted, the cavity pumping plays a relatively minor quantitative role. Therefore we only show two cases, of no-cavity pumping (first row) and high-cavity pumping (second row). As can be seen, there is no strong difference from one spectra with no cavity pumping to its counterpart with large cavity pumping. Third row shows the direct exciton emission that, with no cavity pumping, corresponds to the first row. Indeed, one can observe the richer multiplet structure up to $P_{\sigma} \approx 0.5 \mathrm{~g}$ in the direct exciton emission, whereas only inner peaks are neatly manifest in the cavity emission. This region corresponds to a quantum regime with a few quanta of excitations (and subpoissonian particle number distribution, $g^{(2)}<1$ ) giving rise to clearly resolvable peaks, attributable to the Hamiltonian manifolds. Therefore, a good system (high $Q$ and $g$ ) and a good QD (two-level) emitter suffice to easily and clearly observe quantum effects. There is no need of pumping harder than it has been done in present systems so far. 
In the region $g<P_{\sigma}<30 \mathrm{~g}$, the photon fluctuations are those of a coherent, classical state, $g^{(2)}=1$. Increasing pumping with the intention to penetrate further into the nonlinearity, merely collapses the multiplet structure into a single line, as far as cavity emission is concerned. However, this does not mean that the system is in weak coupling. In the direct exciton emission, the rich SC fine structure has turned into a Mollow triplet [17], that we discuss in depth below. In this region, the first manifolds have crossed to WC but higher manifolds retain SC, bringing the system into lasing. At this point, a change of realm should be performed favoring a classical description. A last transition into thermal light and WC, due to saturation and self-quenching, takes place at $P_{\sigma} \approx 30 g$ that leads to a single central peak in the spectra.

In Fig. 10, we take a closer look into Fig. 9 in the region of the loss of the doublet of inner peaks with increasing electronic pumping, where the system starts to cross from the quantum to the classical regime. In the cavity emission, the doublet of inner peaks collapses into a single line that is going to narrow as the system lases. At the same time, a strikingly richer structure and regime transition is observed in the direct exciton emission. As the peaks are more clearly resolved as explained before [cf. Eqs. (37) and (38)], the "melting" of the Jaynes-Cummings ladder into a classical structure is better tracked down. Indeed, as pumping is increased, broadening of these lines starts to unite them together into an emerging structure of a much less reduced complexity, namely a triplet. In Fig. 11, we provide another zoom of the overall picture given by Fig. 9, this time for the direct exciton emission exclusively. First three rows show the evolution with electronic pumping $P_{\sigma}$ (values in inset) over a wide range of frequencies, up to $\pm 15 \mathrm{~g}$, while the three last rows show the very same spectra, with a one-to-one mapping with previous rows, only in the range of frequencies $\pm 3 g$. The transition manifests to different scales, with a rich fine multiplet structure in the quantum regime, as seen in the zoomed-in region, to a monolithic triplet at higher pumpings, as seen in the enlarged region. On the right, spectra are superimposed to follow their evolution with pumping. The two satellites peaks, at approximately $\pm 2 \sqrt{n_{a}}$, drift apart from the main central one with increasing excitation, and in this sense behave as expected from a Mollow triplet. Various deviations are however observed, of a more or less striking character. The most astonishing feature is the emergence of a very sharp and narrow peak in the center, that has been plotted with its total intensity on the right panel to give a sense of its magnitude. It is clearly seen in the zoomed-region how this peak arises on 
top of the broad mountain of inner peaks, surviving the collapse of the fine structure in the classical regime. This thin central resonance appears when a large truncation is needed. It is a sum of many contributing peaks centered at zero, most of them with very small intensities. This region therefore shows all the signs of a transition from a quantum to a classical system. At low pumping, the inner peaks of all quadruplets coming from low order manifolds are placed approximately at $\pm(\sqrt{n}-\sqrt{n-1}) \neq 0$. Even when they are summed up to produce the total spectrum, the nonlinear doublet is still resolved. At around $P_{\sigma} \approx 1.5 \mathrm{~g}$, manifolds high enough are excited so that for them $\pm(\sqrt{n}-\sqrt{n-1}) \approx 0$. This is a feature of a classical field resulting in a Mollow triplet. Note that nothing of this sort is observed in the cavity emission. The Mollow triplet, whether in atomic physics with coherent excitation or in semiconductor physics with incoherent pumping, is a feature of the quantum emitter itself, when it is directly probed. There is therefore a strong motivation here to detect leak emission of semiconductor structures. The overall features of this "incoherent Mollow triplet" differ from its counterpart namesake in the strong asymmetry of the satellites and their increased broadenings with pumping.

In Fig. 12, we show for Point 2 a similar overall picture as Fig. 9 does for Point 1. Point 2 has larger dissipation and to current estimates, corresponds more closely to the best systems available at the time of writing. As opposed to Point 1, a small cavity pumping has a strong influence on the result, so we display more cases, namely those that range from no cavity pumping (first row) to large cavity pumping $\left(P_{a}=g / 5\right.$, 4th row) with two intermediate cases showing the transfer of the emission from the linear Rabi doublet to the inner peaks arising from transitions between higher manifolds.

The fifth row shows the corresponding direct exciton emission, for the extreme cases of no (1st row) and highest (4th) cavity pumping. The cavity pumping has the important role of revealing the quantum nonlinearity of the system, that was obvious for reference point 1 in any case but is now invisible in the first row, where at increasing electronic pumping, the vacuum Rabi doublet undergoes a rather dull collapse. The same spectra could be expected from a linear (bosonic) model, in the appropriate range of parameters. This is particularly evident in Fig. 13 where three cases of cavity pumping (none, intermediate, and large) are shown for various electronic pumping, both for the cavity and direct emission. Outer lines correspond to zero and inner lines to larger cavity pumping. Note how the intermediate cavity pumping cases display obvious deviation from a bosonic model, that has 

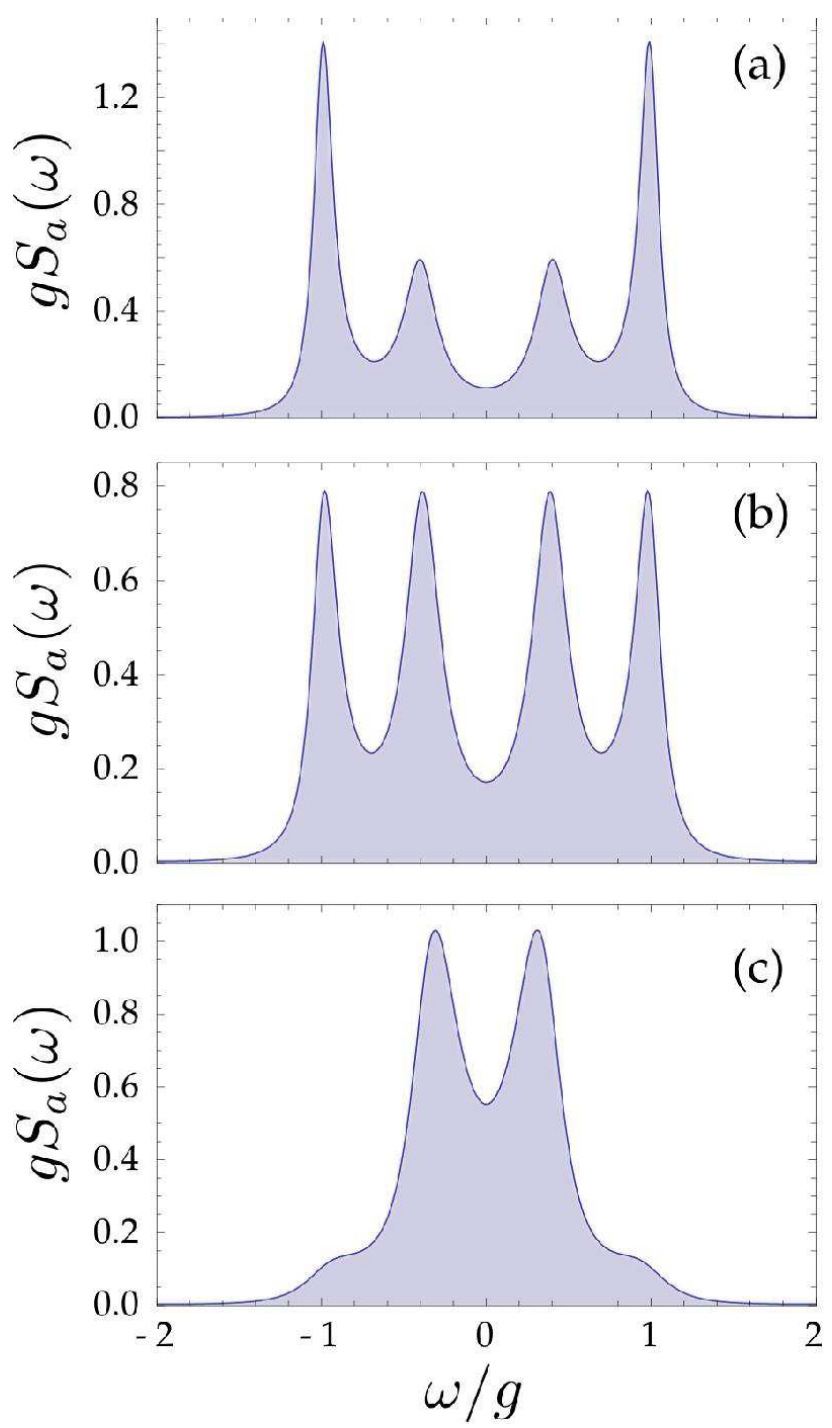

FIG. 7: (Color online). Jaynes-Cummings forks as they appear in the luminescence spectrum of a QD in a microcavity with system parameters given by Point 1 of Fig. 3 and for pumping rates $\left(P_{a}, P_{\sigma}\right) / g$ given by $(\mathrm{a}),(0,0.057) ;(\mathrm{b}),(0.002,0.087)$ and $(\mathrm{c}),(0.001,0.27)$. The two outer peaks at \pm 1 correspond to the vacuum Rabi doublet of the linear limit. Inner peaks correspond to transitions with states of more than one excitation. Although the underlying structure is the same, many variations of the actual lineshapes can be obtained.

essentially the shape of a doublet of Lorentzian peaks (with a dispersive correction that has little bearing on the qualitative aspect of the final result). Cavity pumping literally unravels the nonlinearity. The case of intermediate pumping is the most determining in this aspect as far as cavity emission is concerned, while higher cavity pumpings are more favorable for uncovering quantum features from the direct exciton emission. This is mainly for two 

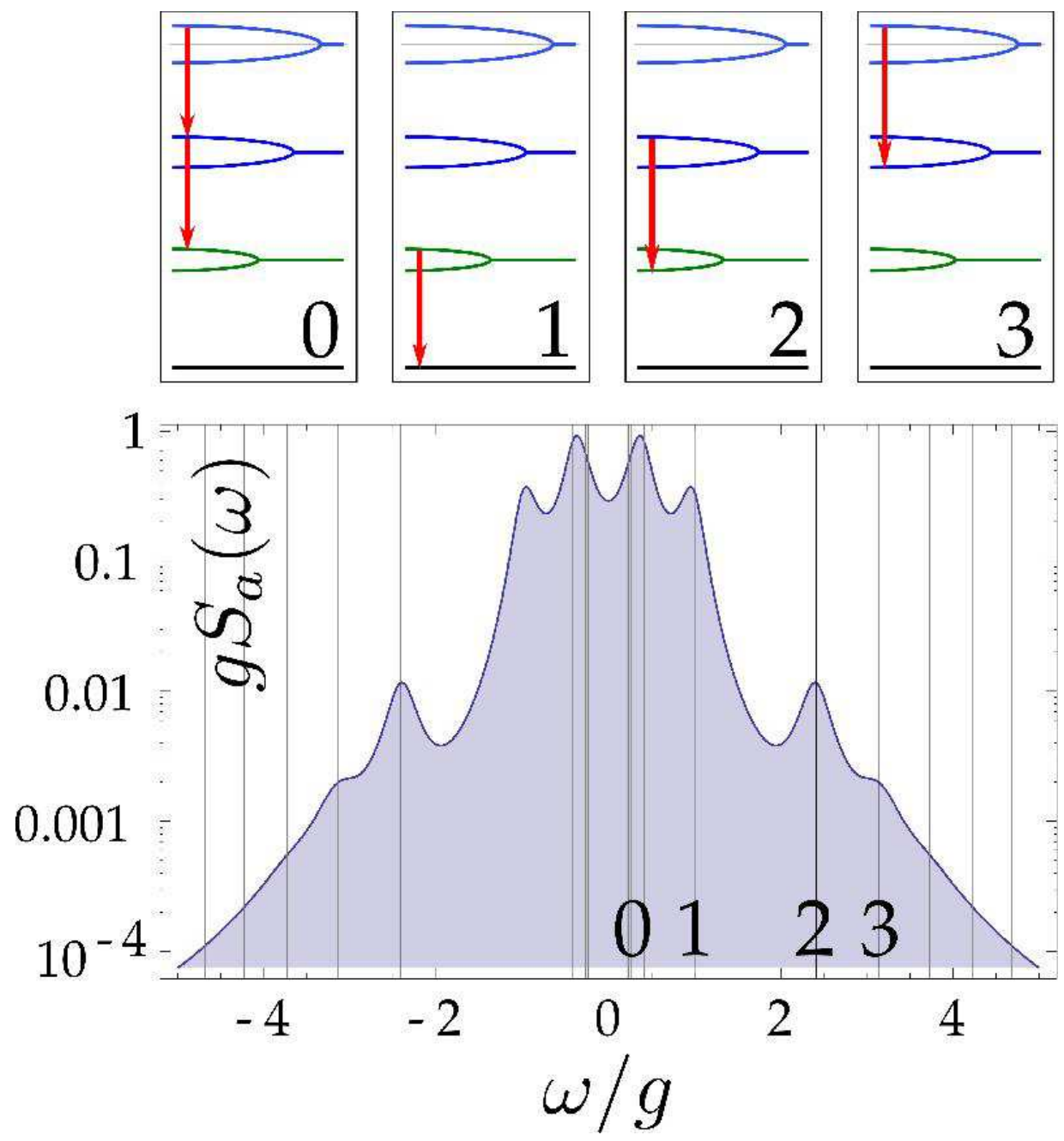

FIG. 8: (Color online). Expanded view in logarithmic scale of a spectrum similar to those of Fig. [7, this time with $\left(P_{a}, P_{\sigma}\right) / g=(0.002,0.076)$. Transitions up to the third manifold (shown in insets) are resolvable. Others are lost in the broadening. The transition energies of the Jaynes-Cummings ladder are shown by vertical lines (up to the third manifold). The Rabi peaks that corresponds to transitions from the first manifold to vacuum (line 1) is in this case dominated by higher transitions that accumulate close to the center (line 0).

reasons. One has to do with the influence of what effective quantum state is realized in the system, that we will discuss in more details in connection with the third reference point. The other being the excitation of higher manifolds from the Jaynes-Cummings ladder, that are now less accessible because of the larger dissipation rates. Note how the disappearance of the vacuum Rabi doublet with increasing $P_{\sigma}$ (with no cavity pumping), is of a different character than for Point 1, where higher $P_{\sigma}$ resulted in an excitation of the upper manifolds 

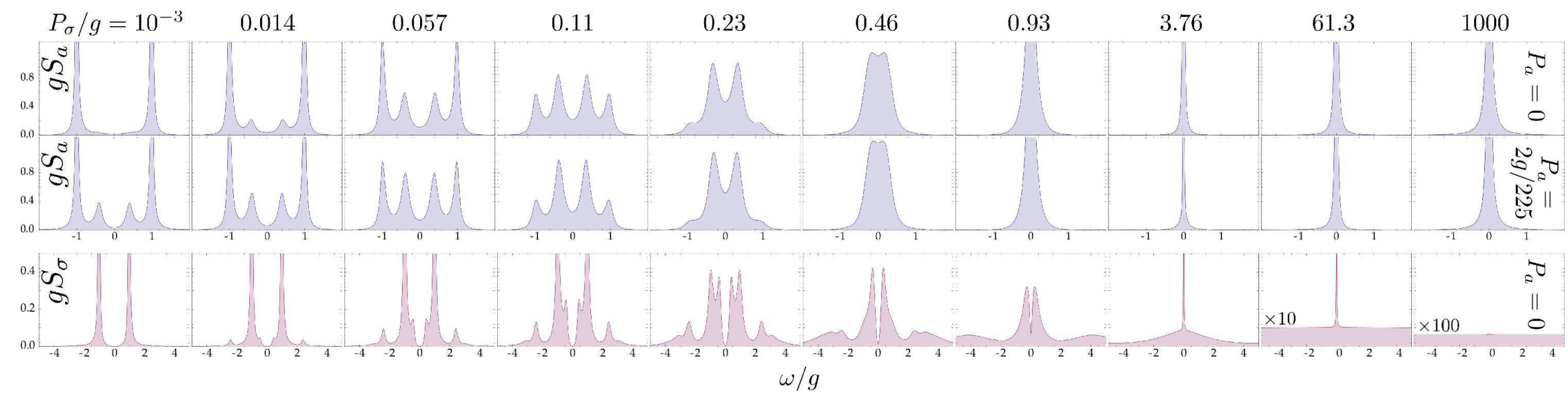

FIG. 9: (Color online). Point 1 of Fig. 3. Spectral emission over a wide range of electronic pumping $P_{\sigma} / g$ from $10^{-3}$ to $10^{3}$ showing the three main regimes: multiplet emission, lasing and quenching. Cavity pumping only affects quantitatively the main features of the emission in this case of very strong coupling, so a small set is shown as representative enough: two upper rows (blue) correspond to cavity emission for no and large cavity pumping, respectively, and lower row (violet) to the direct exciton emission for no cavity pumping. The Jaynes-Cummings fork is clearly resolved at small $P_{\sigma}(<0.2 g)$ and is enhanced by the cavity pumping. At higher electronic pumping $\left(P_{\sigma} \approx 0.5 g\right)$, the multiplet structure collapses into a dominant doublet of inner peaks while the vacuum Rabi peaks melt into its shoulders. Then the system is brought into lasing $\left(g<P_{\sigma}<30 g\right)$ and is finally quenched $\left(P_{\sigma}>30 g\right)$. 


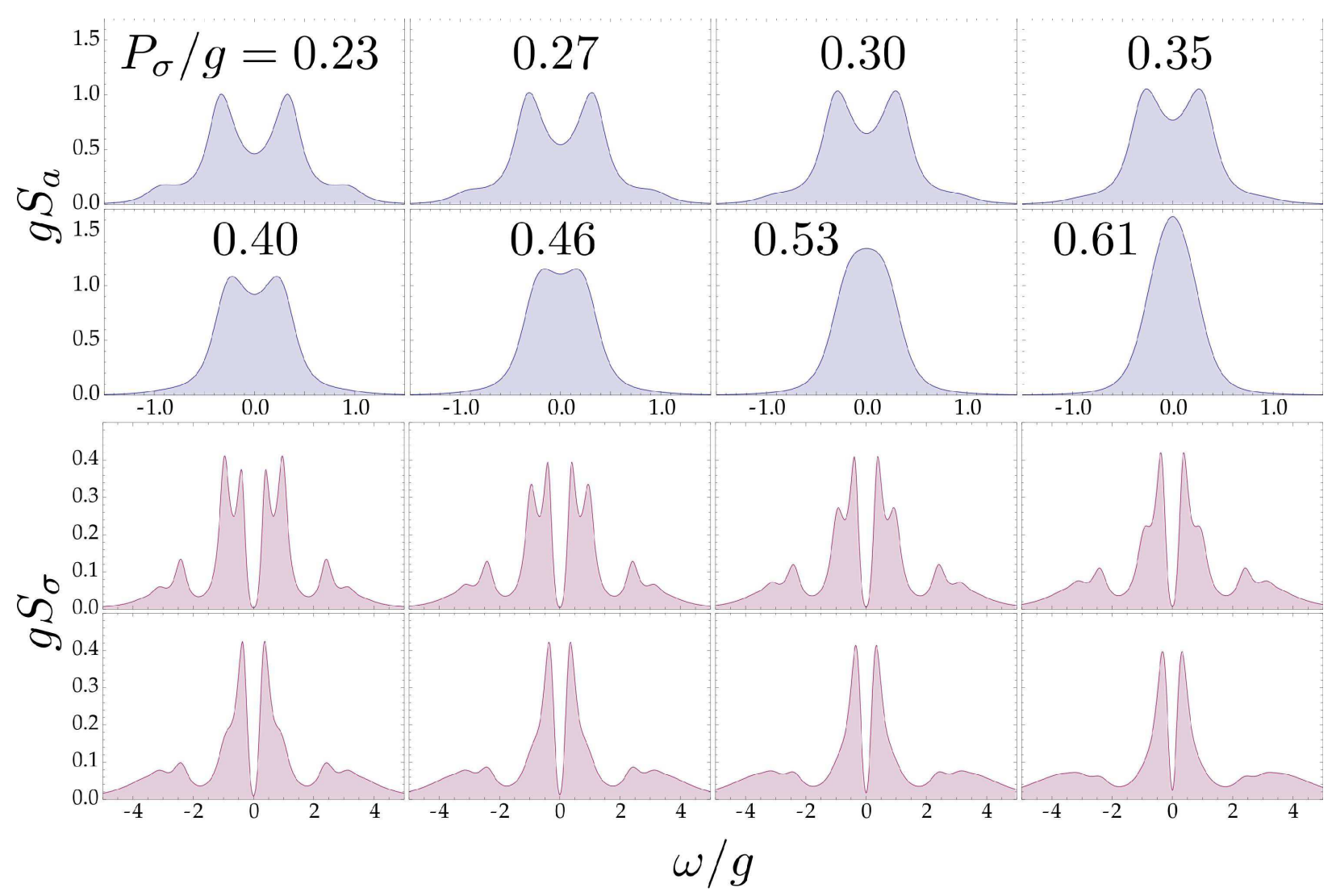

FIG. 10: (Color online). Point 1 of Fig. 3, Details of the loss of the multiplet structure with increasing exciton pumping and zero cavity pumping. The two upper rows (blue) correspond to the cavity emission $S_{a}(\omega)$ and the two lower (violet) to the exciton direct emission $S_{\sigma}(\omega)$. The spectral structure is richer in the exciton spectra that develops a Mollow triplet-like emission.

and a transfer of the dynamics higher in the Jaynes-Cummings ladder, whereas in this case it essentially results in a competition between only the first and second manifold transitions. Cavity pumping can help climbing the ladder with no prejudice to broadening. Finally, even if blurry resolution or statistical noise of an actual experiment would cast doubt on the presence of a quadruplet in such a structure, the transfer with increasing cavity pumping of the emission from outer (linear Rabi) to inner peaks (from the second manifold transitions in this case) makes it clear that the underlying statistics is of a Fermi rather than of a Bose character. In Fig. 14, we show the case $P_{\sigma}=10^{-3} \mathrm{~g}$ for such an increasing cavity pumping for a detailed appreciation of the previous statement. A very close look might still suggest that the case $P_{a}=0$ (outer peaks) still has a small deviation from the linear model that would betray, in a very finely resolved experiment, its non-bosonic or nonlinear character. 


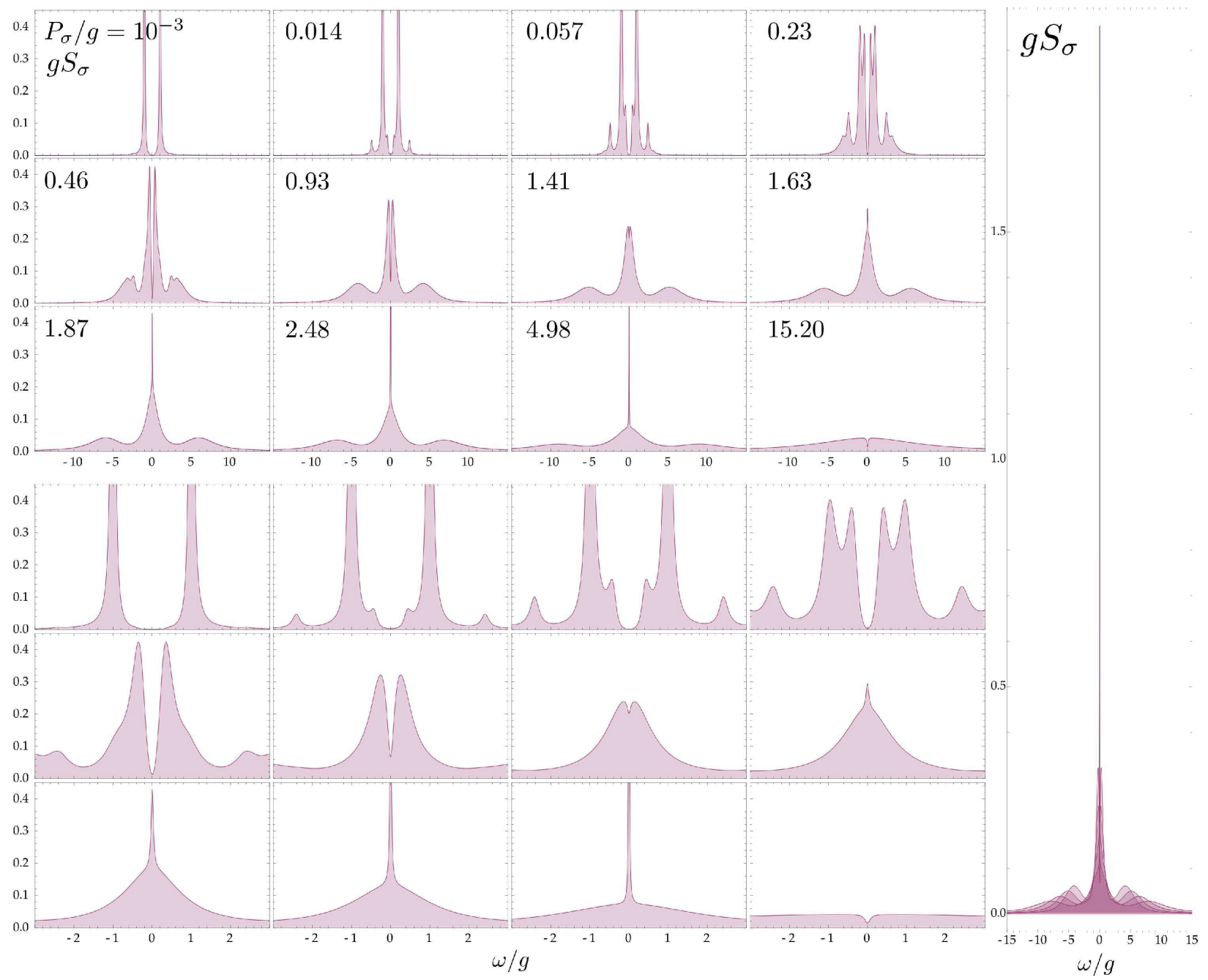

FIG. 11: (Color online). Point 1 of Fig. 3, Incoherent Mollow triplets are observed in the exciton direct emission with broad satellite peaks at approximately $\pm 2 \sqrt{n_{a}}$ and a strong narrow central peak taking over a narrow resonance. Three upper rows show the spectra over the interval $|\omega| \leq 15 g$ allowing to see the satellites. Three lower rows are the same in the window $|\omega| \leq 3 g$, allowing to see the narrow resonance and peak that sit at the origin. Values of the electronic pumping are given in the frame of the first three rows. Cavity pumping is zero but influences very little the Mollow triplets. Rightmost figure superposes various spectra at increasing electronic pumping, showing the drift and broadening of the satellites, and putting to scale the very strong coherent feature at the origin. The incoherent Mollow triplet appears thus very differently from its counterpart under coherent excitation. 
Counter to intuition, this is better seen for vanishing electronic pumping, as otherwise the lines are broadened according to Eq. (10) and this dampens the inner nonlinear peaks. Note, on the other hand, how cavity pumping unambiguously settles the issue.

Finally, we turn to point 3 of Fig. 3, i.e., to the case with high dissipation rates. In this case, as shown in Fig. 15, the Jaynes-Cummings structure is not probed and the spectra are mere Rabi doublets, closing in the WC. These features, by themselves, without a quantitative comparison with other models, say nothing about the nature of the emitter. The linear model, always in SC for this system, leads to a well resolved Rabi doublet in both channels of emission at all pumpings. In the present model, too high a cavity pumping brings the first manifold into WC. In contrast, a small cavity pumping again helps to resolve the Rabi doublet. The main physics at work here is the one that has been amply detailed in part 1 of this study, in the linear case, namely, the effective quantum state realized in the system by the interplay of pumpings and decay. A photon-like quantum state has dispersive corrections that push apart the dressed states (Lorentzians) and therefore enhances the visibility and splitting of the lines. The argument does not adapt itself exactly, for instance line-splitting is not helped or revealed in the exciton emission for most cases, because in this case exciton broadening always spoils resolution of the splitting. In the linear case, there would be a complete symmetry (enhanced splitting in one channel of detection implies reduced visibility in the other channel). This shows again that the same system would lead, in the nonlinear regime, to different results with a bosonic or a fermionic exciton, although both spectra can only feature a doublet or a singlet. A fundamental difference between the models is that the pumps $P_{a}, P_{b}$, always reduce the total broadening of the lines $\left(\Gamma_{+}\right)$while $P_{\sigma}$ increases it. The contribution of pump to the line positions differs greatly from the bosons, as not only $P_{\sigma}$ carries a different sign but also this contribution depends on the manifold. The statistics make also an important difference. Opposite to the wide variety of photon distributions found with a fermion model, cavity and exciton are always in a thermal state for a boson, without quenching or lasing. The issue of the underlying statistics could therefore be settled

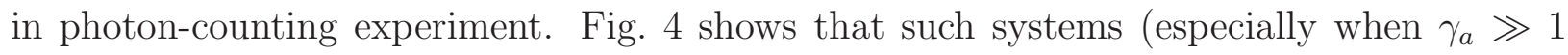
and $\gamma_{\sigma} \rightarrow 0$ ) have the advantage over better cavities that at low electronic pumping and vanishing cavity pumping, the system generates antibunched light, suitable for single-photon emitters (though not on demand). 


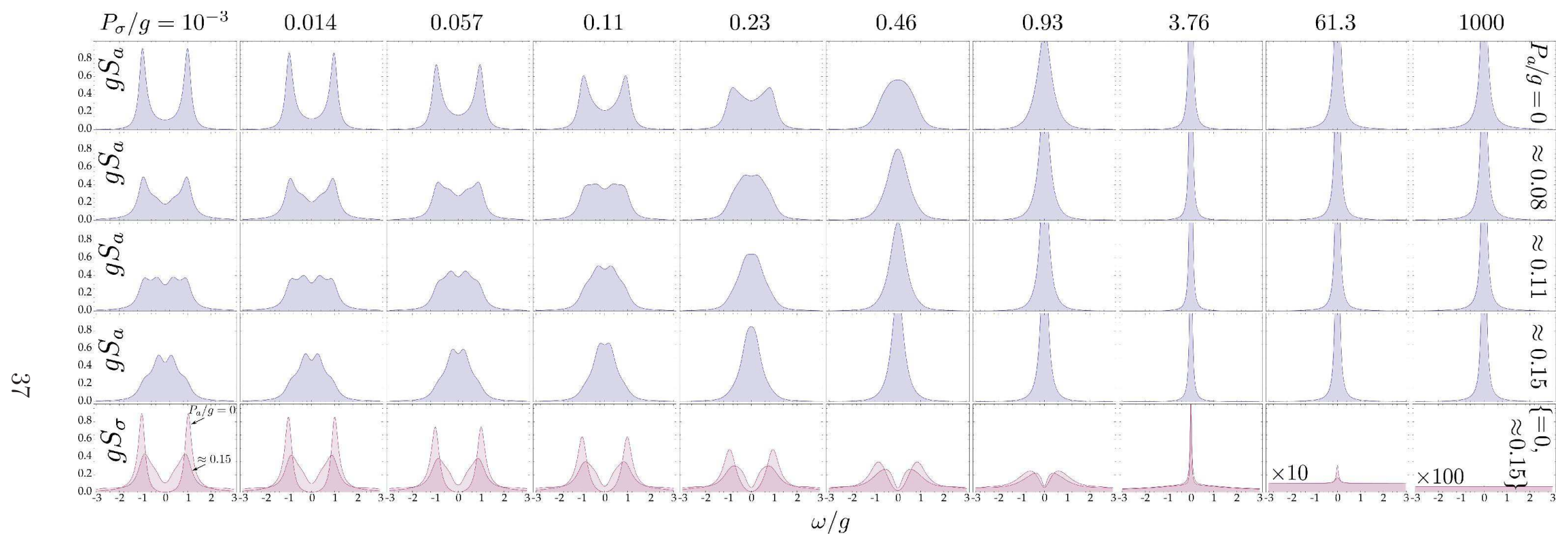

FIG. 12: (Color online). Same as Fig. 9 over the same range of $P_{\sigma}$, but for Point 2 of Fig. 3. In this case, cavity pumping has a strong influence on the cavity luminescence spectra, so we show more cases, namely $P_{a} / g=0$ (upper row), $\approx 0.08$ (second), $\approx 0.11$ (third) and $\approx 0.15$ (fourth) as well as the exciton direct emission spectra $S_{\sigma}$ in the fifth columns with two cases of cavity pumping, $P_{a} / g=0$ (outer peaks) and $\approx 0.15$, corresponding to first and fourth rows of the cavity emission. Exciton spectra are less qualitatively affected by the cavity pumping. With electronic pumping only, no particular feature is observed in the cavity emission. In this case, cavity pumping makes a huge difference by revealing the underlying Jaynes-Cummings ladder. 


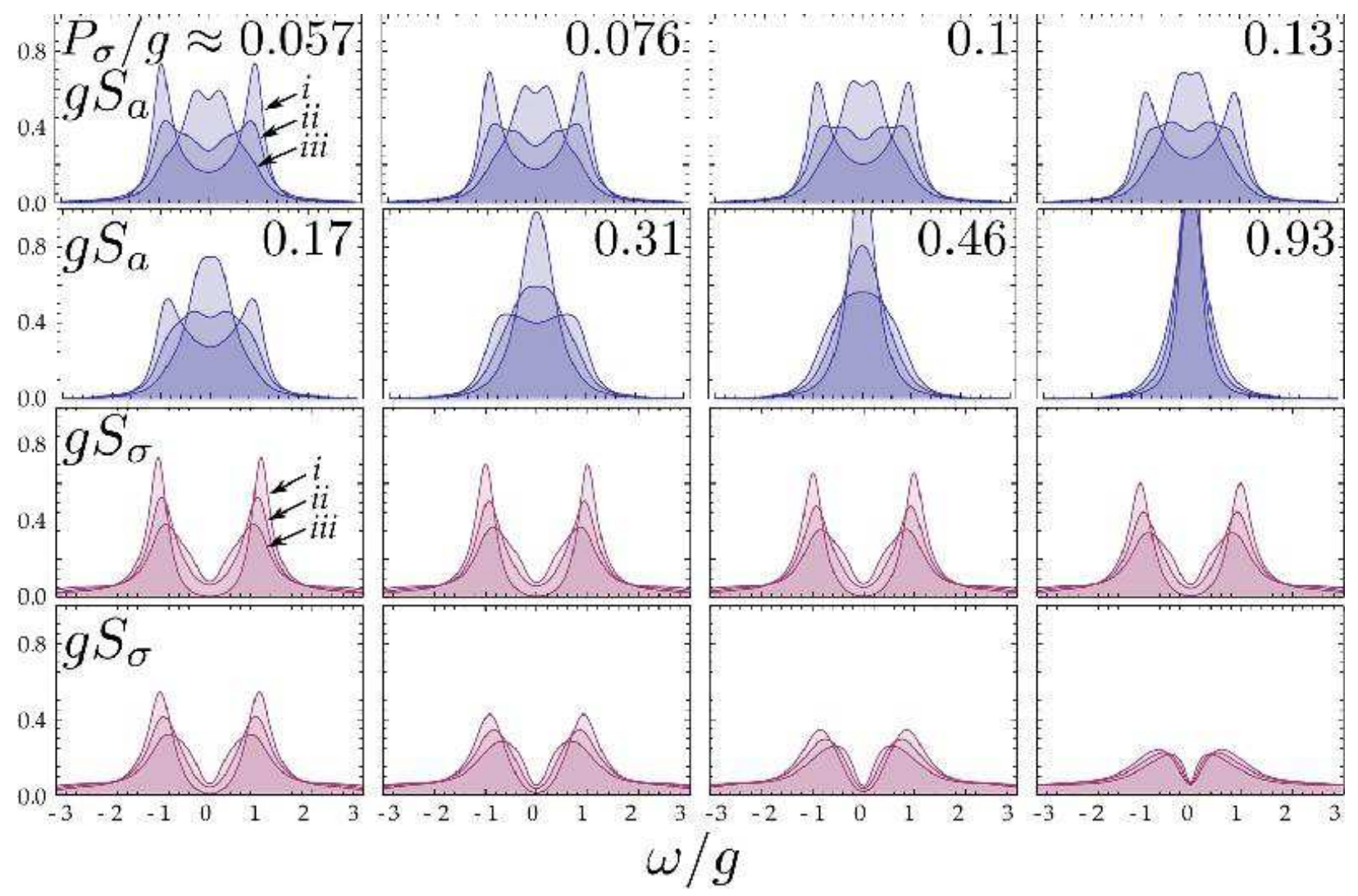

FIG. 13: (Color online). Point 2 of Fig. 3. Details of the loss of the multiplet structure with increasing exciton pumping. Two upper rows (blue) correspond to the cavity emission $S_{a}(\omega)$ and two lower (violet) to the corresponding exciton direct emission $S_{\sigma}(\omega)$ for $P_{a} / g=0(i), \approx 0.076(i i)$ and $\approx 0.15$ (iii) (higher pumping corresponds to innermost peaks). Cavity pumping is essential in such a system to reveal the Fermionic nature of the QD emitter.

\section{LUMINESCENCE SPECTRA WITH DETUNING}

In semiconductors, the detuning between bare modes is a parameter that can easily be varied and which provides useful information of the SC physics. Strong coupling is better studied at resonance, and detuning is mainly used to help locate it, by finding the point where anticrossing is maximum and level repulsion stationary. In a fitting analysis of an experiment, it brings a lot of additional data at the cost of only one additional fitting parameter. In the Fermion case, it also has the benefit of uncovering new qualitative behavior of the PL lineshapes, that are strongly restricted by symmetry at resonance.

Fig. 16 shows the vanishing pumping case of $\omega_{p}$ in Eq. (18) with detuning, i.e., the imaginary part of Eq. (20) for the first row that corresponds to the first manifold (also, 


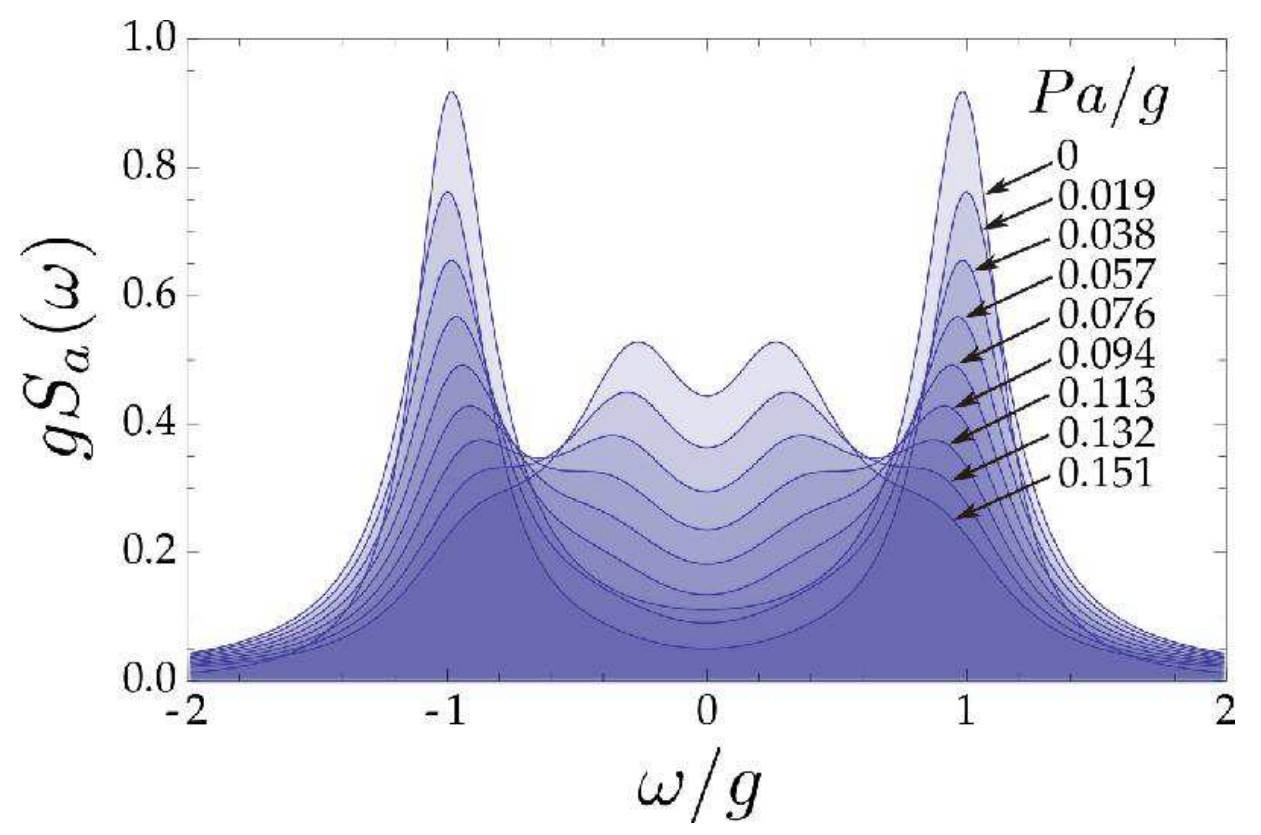

FIG. 14: (Color online). Detail of $S_{a}(\omega)$ for Point 2 of Fig. 3 at vanishing $P_{\sigma}$ for the values of $P_{a} / g$ indicated (higher pumpings correspond to innermost peaks). In a reasonably good QD-cavity system, strong deviations from the linear regime are observed in the emission spectrum, revealing the Jaynes-Cummings fork. The quantum features are made more obvious by increasing the cavity pumping, with a neat renormalization of the dominant doublet even if the quadruplet cannot be resolved experimentally.

the boson case) and of Eq. (21) for the second and third rows, that corresponds to the second and third manifold, respectively. Fourth row is a superposition of all manifolds up to the 15 th one. Detuning is varied in columns, from no detuning (first column) to twice the coupling strength (fifth column). Negative detunings are symmetric with respect to the $x$ axis.

The line opening is common to all manifolds, but note the different behavior of the first manifold (linear or boson case) and higher manifolds: in the first case, one line collapses towards the center (on the cavity mode) while the other recedes away, towards the exciton mode. In the nonlinear case, there is up to four lines, and outer lines are both repelled away while inner lines get both attracted towards the cavity mode, at the center. As we discussed, the total doublet of inner peaks is intense and will dominate. For cases with high dissipation, there is little or no particular insights to be gained from detuning, as, again, most features are lost in broadening. We restrict out attention to Points 1 and 2 in 


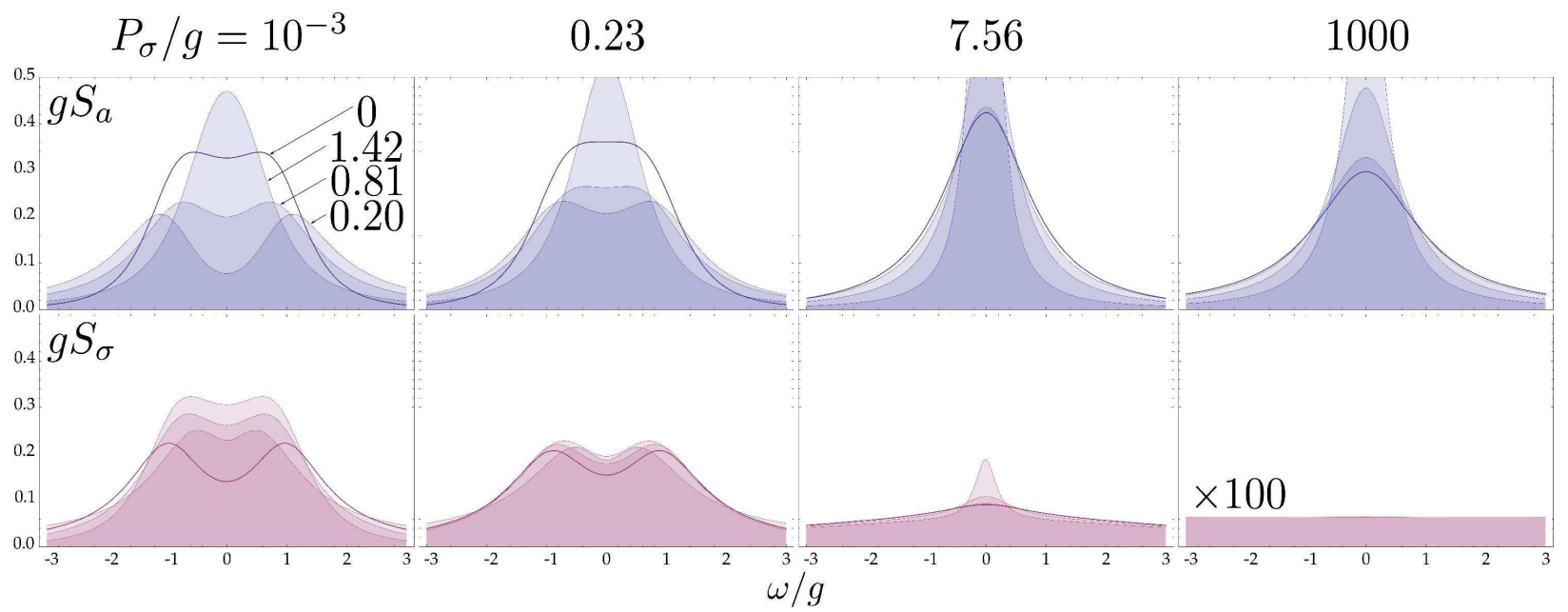

FIG. 15: (Color online). Point 3 of Fig. 3, Spectral emission for the indicated electronic pumping $P_{\sigma} / g: 10^{-3}$ (1st column), $\approx 0.23(2 \mathrm{nd}), \approx 7.56$ (3rd, lasing) and 1000 (4th, quenching), for $P_{a} / g=0$ (thick line with no coloring), $\approx 0.20, \approx 0.81$ and $\approx 1.42$ as indicated in the top left panel, and similarly for others (apart from the case $P_{a}=0$, inner peaks corresponds to higher pumpings). In this system, broadening is always too high to allow any manifestation of the underlying Jaynes-Cummings structure. The structure could be mistaken for a bosonic system (or the other way around). Especially, cavity pumping helps observation of the Rabi doublet in the same way as for bosons modes [1].

what follows. In Fig. 17, PL with detuning are shown for Point 1 in panels (a)-(d) and for Point 2 in panel (e). Panel (d) is a magnified view of panel (a). It is seen clearly how the doublet of inner peaks essentially remains fixed at its resonance position independently of the exciton position. Only at very high detunings does the doublet collapse onto the center. The vacuum Rabi doublet however appears as an anticrossing of the exciton bare mode with the doublet of inner peaks (that eventually becomes the cavity bare mode). Panel (a) is at small electronic pumping and (b), (c) at ten time larger electronic pumping (both no cavity pumping), for the cavity and direct exciton emission, respectively. Again, lower electronic pumping is more prone to reveal rich quantum features. In panel (b) only the inner nonlinear doublet is visible, with a transfer of the emission intensity from one peak (essentially fixed) to the other. The resonance case is plotted in the third panel on first row (third row for the exciton emission) of Fig. 10, The linear Rabi doublet, which trace is seen faintly undergoing anticrossing with the pinned central peaks, provides small shoulders. In general, PL with 
detuning in the Fermi case shows a very characteristic behavior, that cannot be mistaken with a conventional (bosonic) anticrossing experiment.

In panel (e), the case of a more realistic system is shown with detuning. The pinning of the inner peaks is less obvious in this case, although if one draws a vertical line at the resonance, through the minimum of the doublet, one observes that this minimum is fixed. As a result, triplets are obtained in the cavity emission spectra, that are of a very distinct nature than the Mollow triplet observed in the side (exciton) emission of Point 1. The triplets involving the nonlinear doublet are a manifestation of the quantum regime overcoming broadening while the Mollow triplet is a manifestation of the lasing regime.
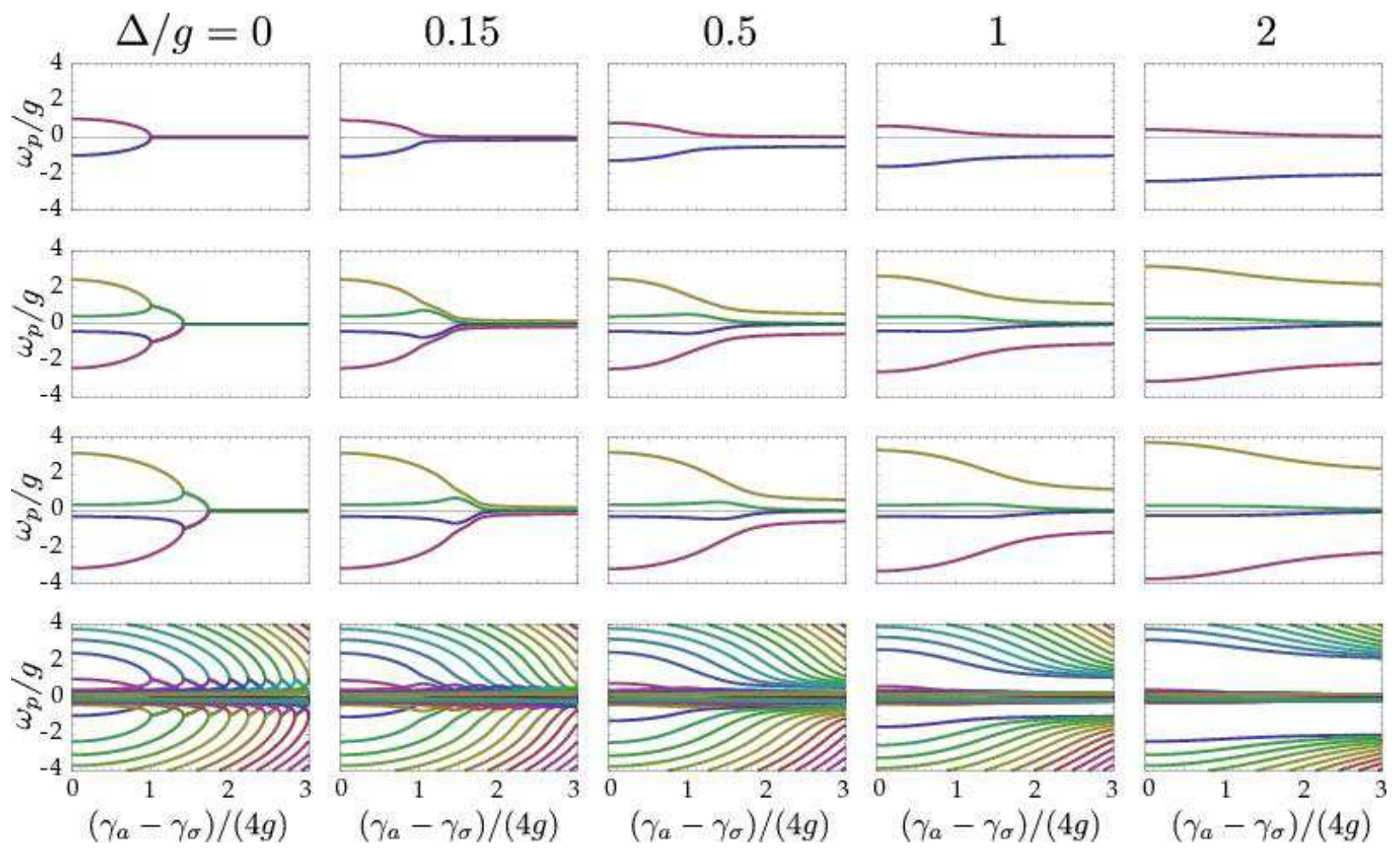

FIG. 16: (Color online). Positions $\omega_{p} / g$ of the lines around $\omega_{a}=0$ in the luminescence spectrum with detuning and in the absence of pump. Columns correspond to various detunings, first column being the case of resonance (cf. Fig. 2). First three rows show in isolation the first, second and third manifold, respectively. First manifold corresponds to the Boson or linear case [1]. Fourth row shows all manifolds together. Left-bottom panel is detailed for positive $\omega_{p}$ in Fig. 2 , 

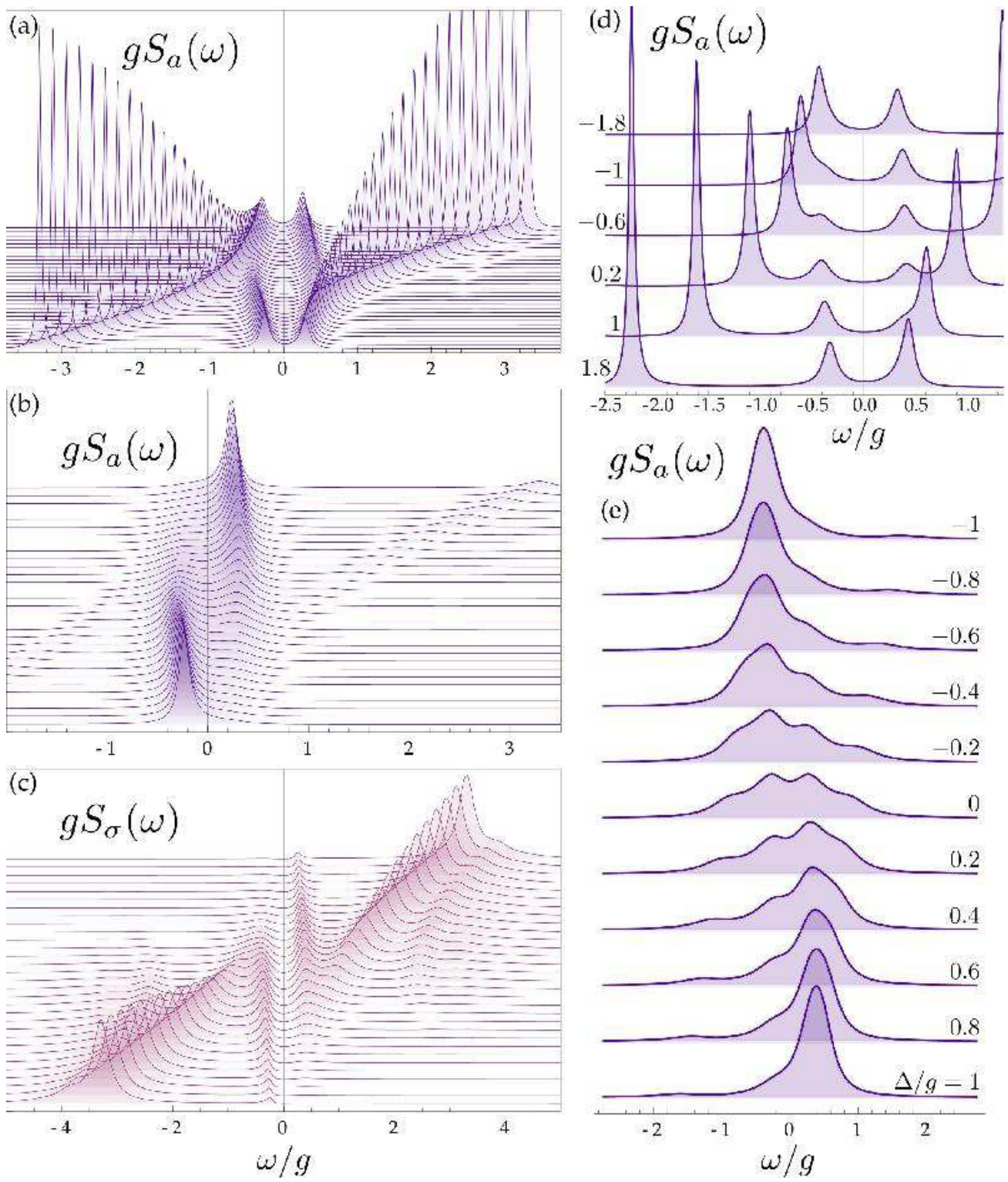

FIG. 17: (Color online). Anticrossing of the luminescence lines as detuning $\Delta=\omega_{a}-\omega_{\sigma}$ is varied. Here, $\omega_{a}=0$ is fixed and the QD bare energy is tuned from below the cavity (positive detuning) to above (negative detuning). Panels (a)-(d) correspond to Point 1 and panel (e) to Point 2. (a)-(d) are at zero cavity pumping, $P_{a}=0$. (a) and (d) are for $P_{\sigma}=0.03 g[(\mathrm{~d})$ is a zoom of (a)] and (b)-(c) for $P_{\sigma}=0.3 g$. (a), (b), (d) are the cavity emission $S_{a}(\omega)$ and (c) the direct exciton emission $S_{\sigma}(\omega)$. (e) is for $P_{\sigma}=10^{-3} g$ and $P_{a}=g / 5$ (cf., 7th row, 1st column of Fig. 12). The nonlinear central peaks give rise to very characteristic anticrossing profiles. 


\section{DISCUSSION AND CONCLUSIONS}

We now give an overview of our results on the spectral lineshapes of a QD that obeys Fermi statistics, in SC with the single mode of a semiconductor microcavity, and extend our previous exposition with a more general discussion. The main results of our analysis are as follow.

Manifestations of nonlinearities in the SC physics of a genuine quantum emitter are, counter to naive expectations, no better sought at high pumpings, looking forward to large number of excitations. The quantum regime involves a few quanta only. It is achieved and better manifests with low pumpings in high quality samples (meaning that the excitonphoton coupling should be much higher than the decays and dissipation rates). Higher pumpings will cross to the classical regime where the quantumness of the system - that manifests with clearly separated peaks attributable to well identified transitions in the JaynesCummings ladder - will give over to a very large number of very small correlators, conveying that a continuous field is taking over quantum discretization. High pumping that bring the system into a lasing regime has a behavior that is better expressed by classical fields and classical physics. The crossover from a quantum to a classical description is an interesting problem that such a model - the dissipative Jaynes-Cummings Hamiltonian with incoherent pumping-seems particularly well suited to track theoretically.

Cavity pumping is an important factor to take into account. First because of its relevance in an actual experiment, where it can arise due to secondary effects such as other dots (not in $\mathrm{SC}$ ) emitting in the cavity, temperature, or a variety of other factors. It could conceivably also be input directly by the experimentalist. Cavity pumping has many virtues for the physics of SC in a semiconductor. Because the typical type of excitation is electronic and the typical channel of detection is photonic, $\mathrm{SC}$ is hampered as compared to the microwave cavity case where detection and excitation are on the same footing (both directly on the atom). A cavity pumping can help balance this situation and provide an effective photon character to the states realized in the semiconductor, enhancing or even revealing spectral structures. This phenomena manifests also in the Boson case and has been investigated and explained in its full detailed in part I of this work. Also in the Fermion case, cavity pumping is beneficial for the same reasons, and it can help go beyond the linear regime (with a Rabi doublet) to the nonlinear quantum regime, typically by making emerge additional 
quadruplets of the Jaynes-Cummings, with a doublet of inner peaks to be sought as the strongest signature.

The counterpart of a Mollow triplet is observed in the best samples, in the direct exciton emission. It features a narrow resonance in the center of the spectrum that turns into a sharp emission line. The incoherent Mollow triplet is a striking manifestation of a crossover from the quantum to the classical limit, with a series of many peaks easily identifiable with dressed quantum states, melting into a monolithic structure of reduced complexity (a triplet) with no identifiable contributions from separable processes. When the Mollow triplet is fully formed, the cavity mode is in the lasing regime. The Mollow triplet is lost as the system is quenched with no return to quantum behaviors. This provides the general sequence of regimes with increasing electronic pumping: quantum regime, lasing (classical) regime and quenched (also classical but thermal) regime.

\section{Acknowledgments}

Most numerical computations presented here have been performed on the Iridis cluster of the University of Southampton, for which authors gratefully acknowledge the support from Dr. Ivan Woulton. This work has been supported by the Spanish MEC under contracts Consolider-Ingenio2010 CSD2006-0019, MAT2005-01388 and NAN2004-09109-C04-3 and by CAM under contract S-0505/ESP-0200. EdV acknowledges support of the FPU from the Spanish MEC.

[1] F. P. Laussy, E. del Valle, and C. Tejedor, arXiv:0807.3194 (2008).

[2] E. Jaynes and F. Cummings, Proc. IEEE 51, 89 (1963).

[3] F. P. Laussy, M. M. Glazov, A. Kavokin, D. M. Whittaker, and G. Malpuech, Phys. Rev. B 73, $115343(2006)$.

[4] S. Rudin and T. L. Reinecke, Phys. Rev. B 59, 10227 (1999).

[5] Y. Zhu, D. J. Gauthier, S. E. Morin, Q. Wu, H. J. Carmichael, and T. W. Mossberg, Phys. Rev. Lett. 64, 2499 (1990).

[6] L. Schneebeli, M. Kira, and S. W. Koch, Phys. Rev. Lett. 101, 097401 (2008).

[7] J. T. Steiner, M. Kira, and S. W. Koch, Phys. Rev. B 77, 165308 (2008). 
[8] K. Srinivasan and O. Painter, Nature 450, 862 (2007).

[9] D. Press, S. Götzinger, S. Reitzenstein, C. Hofmann, A. Löffler, M. Kamp, A. Forchel, and Y. Yamamoto, Phys. Rev. Lett. 98, 117402 (2007).

[10] M. Kroner, A. O. Govorov, S. Remi, B. Biedermann, S. Seidl, A. Badolato, P. M. Petroff, W. Zhang, R. Barbour, B. D. Gerardot, et al., Nature 451, 311 (2008).

[11] J. J. Sanchez-Mondragon, N. B. Narozhny, and J. H. Eberly, Phys. Rev. Lett. 51, 550 (1983).

[12] J. I. Inoue, T. Ochiai, and K. Sakoda, Phys. Rev. A 77, 015806 (2008).

[13] H. J. Carmichael, R. J. Brecha, M. G. Raizen, H. J. Kimble, and P. R. Rice, Phys. Rev. A 40, 5516 (1989).

[14] L. C. Andreani, G. Panzarini, and J.-M. Gérard, Phys. Rev. B 60, 13276 (1999).

[15] G. Cui and M. G. Raymer, Phys. Rev. A 73, 053807 (2006).

[16] A. Auffèves, B. Besga, J.-M. Gérard, and J.-P. Poizat, Phys. Rev. A 77, 063833 (2008).

[17] B. R. Mollow, Phys. Rev. 188, 1969 (1969).

[18] C. M. Savage, Phys. Rev. Lett. 63, 1376 (1989).

[19] H. Freedhoff and T. Quang, Phys. Rev. Lett. 72, 474 (1994).

[20] A. Barchielli and N. Pero, J. Opt. B 4, 272 (2002).

[21] L. Florescu, Phys. Rev. A 74, 063828 (2006).

[22] M. Bienert, J. M. Torres, S. Zippilli, and G. Morigi, Phys. Rev. A 76, 013410 (2007).

[23] J. P. Clemens and P. R. Rice, Phys. Rev. A 61, 063810 (2000).

[24] J. I. Perea, D. Porras, and C. Tejedor, Phys. Rev. B 70, 115304 (2004).

[25] M. Löffler, G. M. Meyer, and H. Walther, Phys. Rev. A 55, 3923 (1997).

[26] J. P. Clemens, P. R. Rice, and L. M. Pedrotti, J. Opt. Soc. Am. B 21, 2025 (2004).

[27] T. B. Karlovich and S. Y. Kilin, Laser Phys. 103, 280 (2007).

[28] T. B. Karlovich and S. Y. Kilin, Laser Phys. 18, 783 (2008).

[29] K. Hennessy, A. Badolato, M. Winger, D. Gerace, M. Atature, S. Gulde, S. Fălt, E. L. Hu, and A. Ǐmamō̄lu, Nature 445, 896 (2007).

[30] T. Feldtmann, L. Schneebeli, M. Kira, and S. W. Koch, Phys. Rev. B 73, 155319 (2006).

[31] C. Gies, J. Wiersig, M. Lorke, and F. Jahnke, Phys. Rev. A 75, 013803 (2007).

[32] H. J. Carmichael, Statistical methods in quantum optics 1 (Springer, 2002), 2nd ed.

[33] E. del Valle, F. P. Laussy, F. Troiani, and C. Tejedor, Phys. Rev. B 76, 235317 (2007).

[34] G. Khitrova, H. M. Gibbs, M. Kira, S. W. Koch, and A. Scherer, Nature Phys. 2, 81 (2006). 
[35] F. P. Laussy, E. del Valle, and C. Tejedor, Phys. Rev. Lett. 101, 083601 (2008).

[36] O. Benson and Y. Yamamoto, Phys. Rev. A 59, 4756 (1999).

[37] Y. Mu and C. M. Savage, Phys. Rev. A 46, 5944 (1992).

[38] C. Ginzel, H.-J. Briegel, U. Martini, B.-G. Englert, and A. Schenzle, Phys. Rev. A 48, 732 (1993).

[39] B. Jones, S. Ghose, J. P. Clemens, P. R. Rice, and L. M. Pedrotti, Phys. Rev. A 60, 3267 (1999).

[40] T. B. Karlovich and S. Y. Kilin, Opt. Spectrosc. 91, 343 (2001).

[41] A. Kozlovskii and A. Oraevskii, Sov. Phys. JETP 88, 666 (1999).

[42] M. O. Scully and M. S. Zubairy, Quantum optics (Cambridge University Press, 2002). 